Supporting Information for:

\title{
A Biradical Balancing Act: Redox Amphoterism in a Diindenoanthracene Derivative Results from Quinoidal Acceptor and Aromatic Donor Motifs
}

Gabriel E. Rudebusch, ${ }^{\dagger}$ Guzmán L. Espejo ${ }^{\ddagger}$ José L. Zafra ${ }^{\ddagger}$ Miriam Peña-Alvarez, ${ }^{\ddagger}$ Sarah N. Spisak, Kotaro Fukuda," Zheng Wei, ${ }^{\S}$ Masayoshi Nakano, ${ }^{\text {, }}$ Marina A. Petrukhina, ${ }^{*}$ Juan Casado, ${ }^{*,+}$ and Michael M. Haley,,+

Department of Chemistry \& Biochemistry and the Materials Science Institute, University of Oregon, Eugene, Oregon 97403-1253, United States

DDepartment of Physical Chemistry, University of Malaga, Campus de Teatinos s/n 229071 Malaga, Spain

${ }^{\S}$ Department of Chemistry, University at Albany, State University of New York, Albany, New York 12222-0100, United States

"Department of Materials Engineering Science, Graduate School of Engineering Science, Osaka University, Toyonaka, Osaka 560-8531, Japan

Table of Contents page

Variable Temperature UV-vis Spectroscopy

Spectroelectrochemistry

Experimental Details for Chemical Reduction

X-ray Crystallography

High Pressure Measurements

Computational Details

S14

Cartesian Coordinates

S16

TDDFT Computational Details

S31

Hirschfield Charges

S36

References

S39 
Variable Temperature UV-vis Spectroscopy

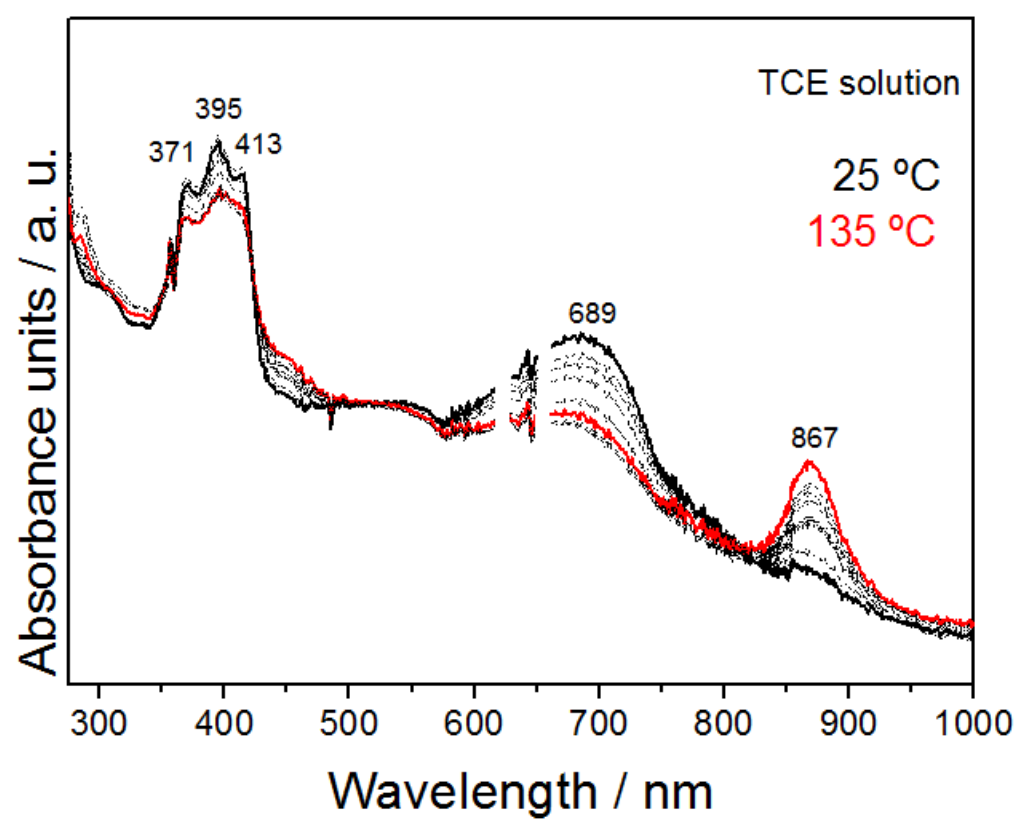

Figure S1. DIAn in tetrachloroethane from 25 to $135{ }^{\circ} \mathrm{C}$. The $689 \mathrm{~nm}$ peak corresponding to neutral DIAn is fully recovered upon cooling to $25^{\circ} \mathrm{C}$.

\section{Spectroelectrochemistry}

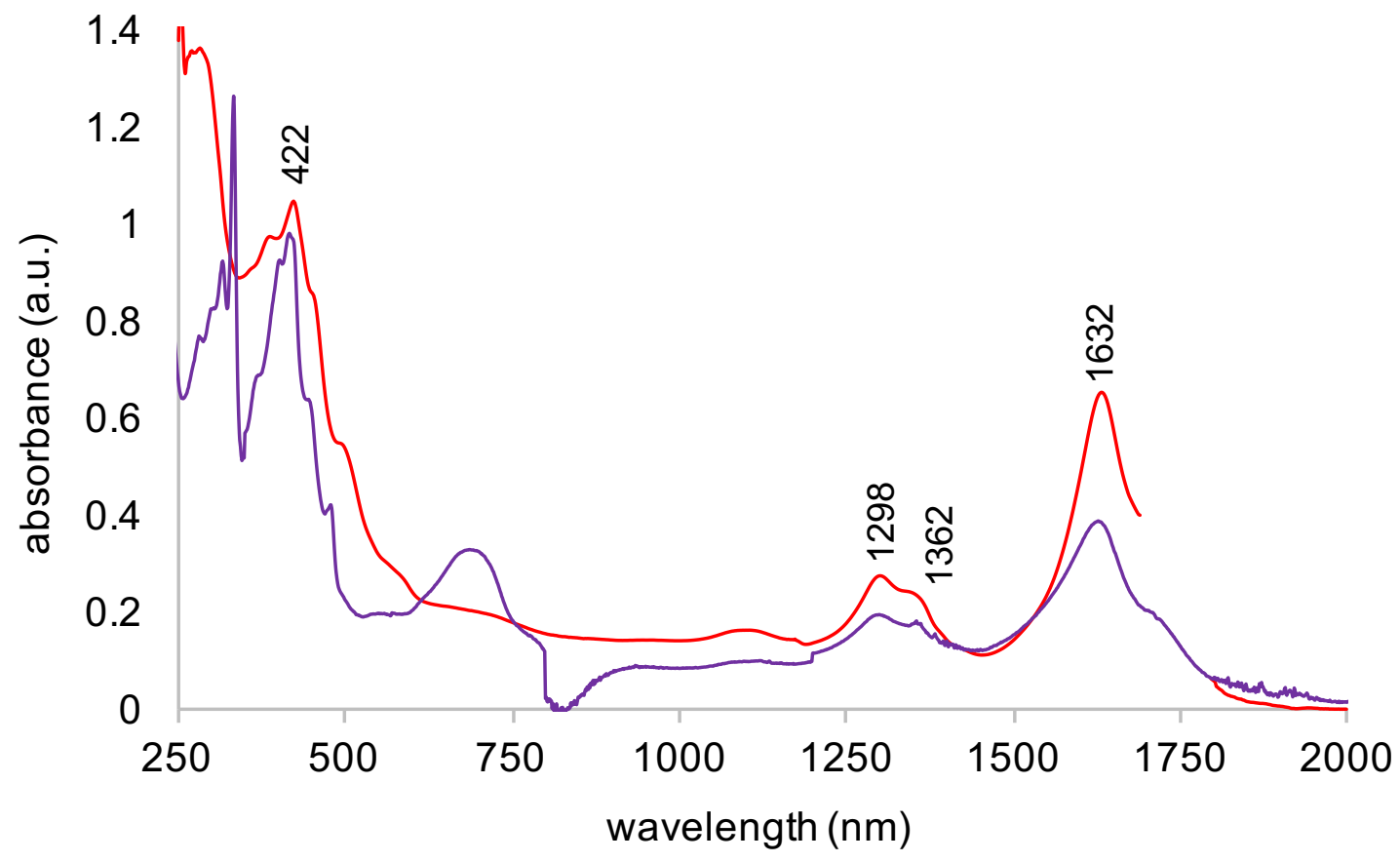

Figure S2. Comparison of DIAn*- (red) generated by contact with potassium in THF and by electrolysis (violet). 


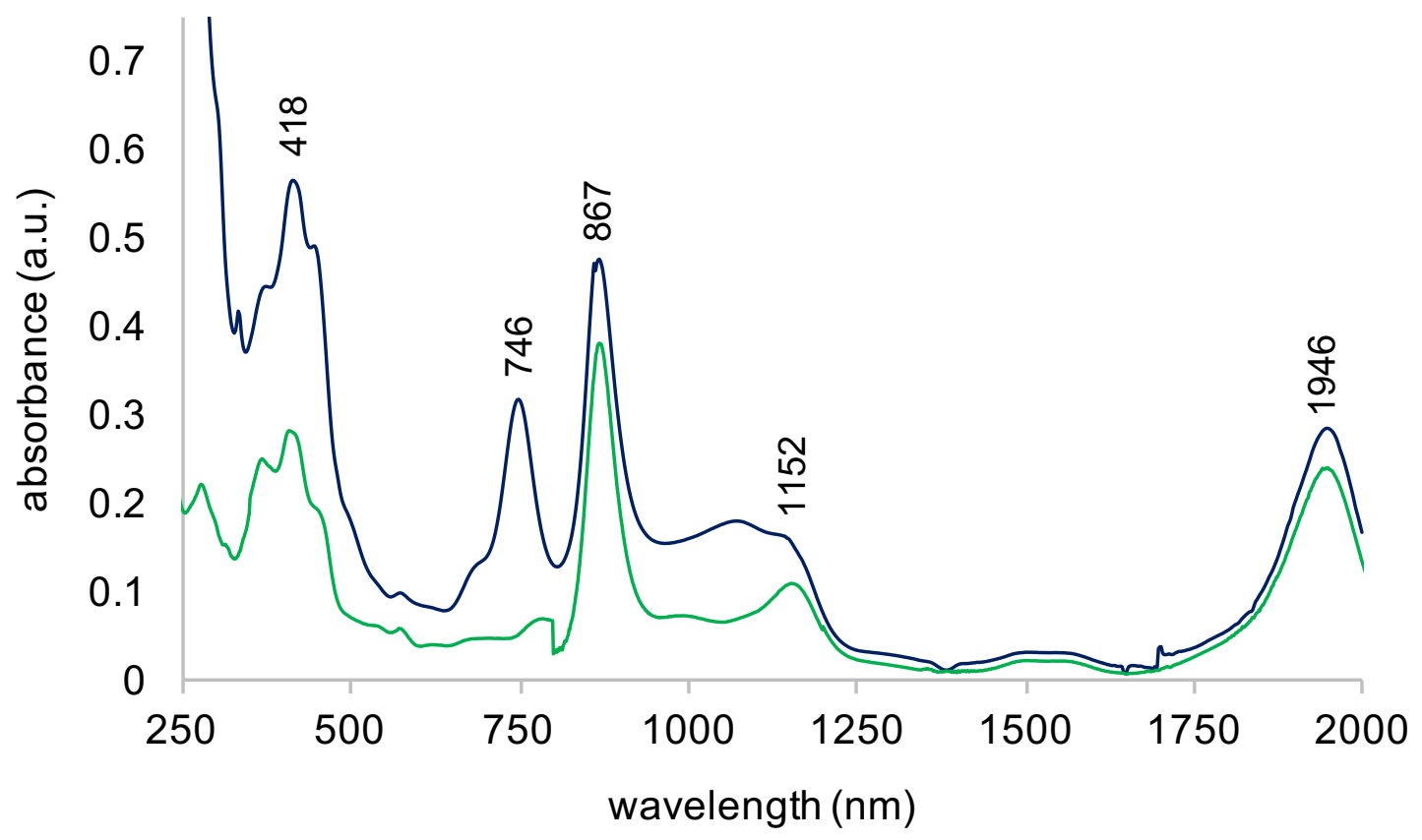

Figure S3. Comparison of DIAn*+ (blue) generated by $\mathrm{NOBF}_{4}$ in $\mathrm{CH}_{2} \mathrm{Cl}_{2}$ and by electrolysis (green). The absorbance at $746 \mathrm{~nm}$ could be due to over-oxidation towards DIAn ${ }^{2+}$.

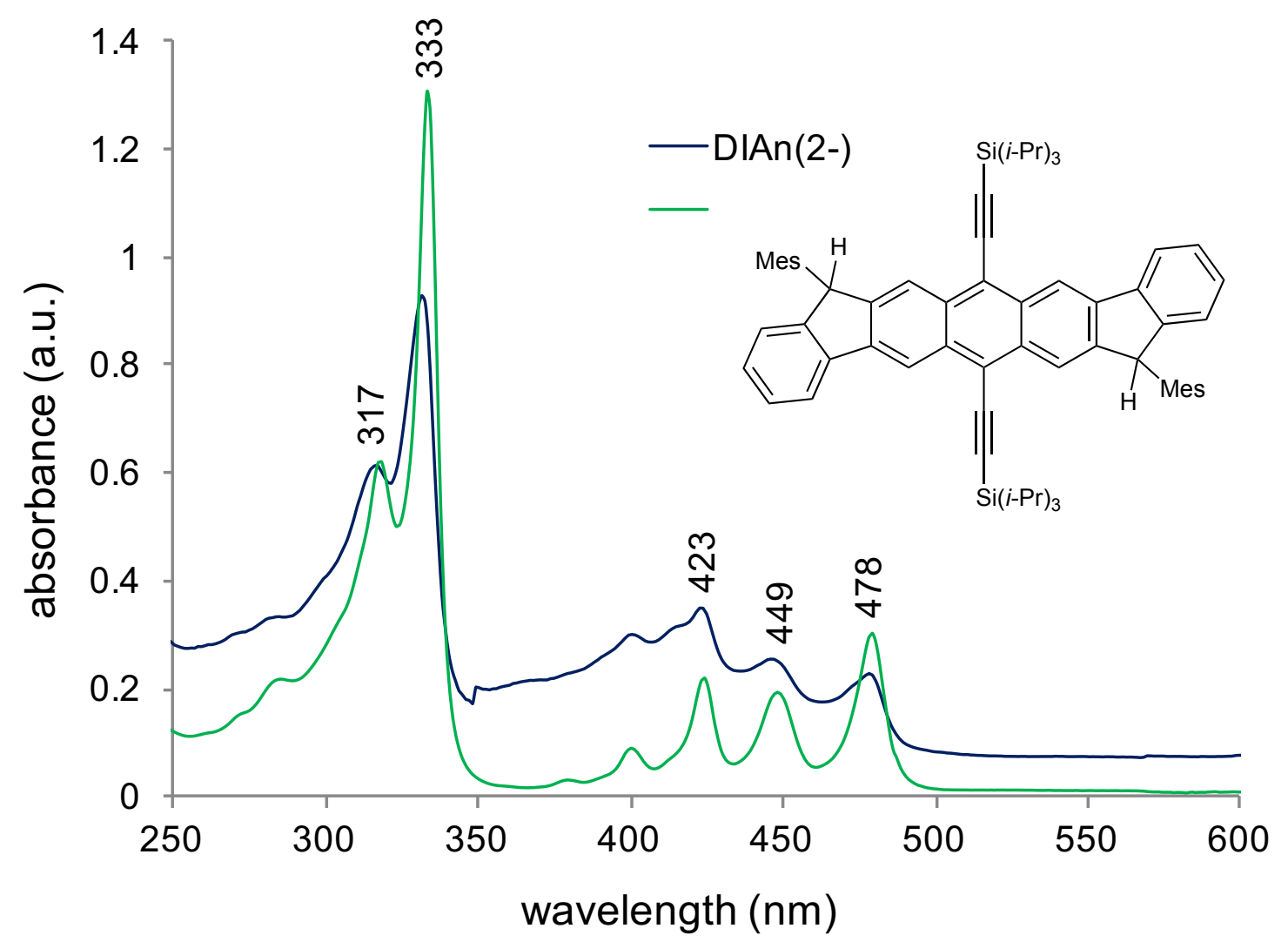

Figure S4. Comparison of DIAn ${ }^{2-}$ prepared by electrolysis (navy) and the dihydro-DIAn precursor (green). 


\section{Experimental Details for Chemical Reduction}

All manipulations were carried out using break-and-seal ${ }^{1}$ and glove-box techniques under an atmosphere of argon. THF and hexanes were dried over Na/benzophenone and distilled prior to use. THF$d_{8}$ and diglyme were dried over sodium-potassium alloy and distilled prior to use. Potassium was purchased from Strem Chemicals. 18-Crown-6 ether was purchased from Sigma Aldrich and dried over $\mathrm{P}_{2} \mathrm{O}_{5}$ in vacuo for $24 \mathrm{~h}$. DIAn was prepared as described previously. ${ }^{2}$

\section{$\left[\left\{\mathrm{K}\left(18\right.\right.\right.$-crown-6) $\left.\left.(\mathrm{THF})_{2}\right\} \mathrm{DIAn}{ }^{\cdot-}\right] \cdot 2 \mathrm{THF}($ Compound 1)}

THF (3 mL) was added to a closed system containing DIAn ( 2 mg), excess of $\mathrm{K}$ metal and 18crown-6. The initial color of the mixture was blue-green (neutral parent ligand). After stirring at room temperature for $10 \mathrm{~min}$, the reaction solution turned deep green. The mixture was stirred at room temperature until brown swirls were seen coming off the $\mathrm{K}$ metal (indication of the dianion beginning to form). The mixture was then filtered and the green filtrate was layered with hexanes $(3.1 \mathrm{~mL})$ and cooled to $0{ }^{\circ} \mathrm{C}$. After $5 \mathrm{~d}$, dark brown plates of 1 were formed in moderate yield (ca. $40 \%$ ). Crystals were collected, washed with hexanes $(3 \times 2 \mathrm{~mL})$ and dried before measurements.

\section{$\left[\left\{\mathrm{K}\left(18 \text {-crown-6) }(\mathrm{THF})_{2}\right\}_{2} \mathrm{DIAn}^{2-}\right] \cdot 4 \mathrm{THF}(\mathrm{Compound} 2)\right.$}

THF (3 mL) was added to a closed system containing DIAn ( 3 mg), excess of $\mathrm{K}$ metal and 18crown-6. The initial color of the mixture was blue-green. After being stirred at room temperature for 10 min, the reaction solution turned deep green. The mixture was stirred for an additional $2 \mathrm{~h}$ resulting in a brown suspension. After stirring at room temperature for a total of $10 \mathrm{~h}$, the deep brown mixture was filtered and the orange-brown filtrate was layered with hexanes $(2.7 \mathrm{~mL})$ and cooled to $10{ }^{\circ} \mathrm{C}$. Red-brown plate shaped crystals of 2 had formed in good yield ( $c a .70 \%$ ) after 3 d. Crystals were collected, washed with hexanes $(3 \times 2 \mathrm{~mL})$ and dried before measurements.

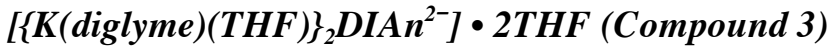

THF (3 mL) was added to a closed system containing DIAn $(\sim 2 \mathrm{mg})$ and an excess of K metal. The initial color of the mixture was blue-green. After being stirred at room temperature for 10 min, the reaction solution turned deep green. The mixture was stirred for an additional $2 \mathrm{~h}$ resulting in a brown suspension. After stirring at room temperature for a total of $10 \mathrm{~h}$, the deep brown mixture was filtered and the brown filtrate was layered with diglyme:hexanes $(1: 20,1.4 \mathrm{~mL})$. The ampoule was placed at $0{ }^{\circ} \mathrm{C}$. After $4 \mathrm{~d}$, orange-brown plate-shaped crystals of $\mathbf{3}$ had formed in moderate yield ( $c a .35 \%)$. Crystals were collected, washed with hexanes $(3 \times 2 \mathrm{~mL})$ and dried before measurements. 


\section{X-ray Crystallography}

Data collections for 1, 2, and $\mathbf{3}$ were performed on a Bruker D8 VENTURE X-ray diffractometer with PHOTON $100 \mathrm{CMOS}$ detector and mirror-monochromated $\mathrm{Cu}-\mathrm{K} \alpha$ radiation $(\lambda=1.54178 \AA)$ at $T=$ 100(2) K. Data were corrected for absorption effects using the empirical method SADABS. ${ }^{3}$ The structures were solved by direct methods and refined using the Bruker SHELXTL (Version 6.14) software package. ${ }^{4}$ All non-hydrogen atoms were refined anisotropically. All $\mathrm{H}$-atoms were included at calculated positions and refined as riders, with $\mathrm{U}_{\mathrm{iso}}(\mathrm{H})=1.2 \mathrm{U}_{\mathrm{eq}}(\mathrm{C})$ and $\mathrm{U}_{\mathrm{iso}}(\mathrm{H})=1.5 \mathrm{U}_{\mathrm{eq}}(\mathrm{C})$ for methyl groups. In $\mathbf{1}$, two of three isopropyl groups connected with silicon atom were found to be disordered. The disorder was modelled with two orientations having relative occupancies of 0.70:0.30 and 0.53:0.47 for the two parts, separately. The geometries of the disordered parts were restrained to be similar. The anisotropic displacement parameters of the disordered molecules in the direction of the bonds were restrained to be equal with a standard uncertainty of $0.01 \AA^{2}$. They were also restrained to have the same $\mathrm{U}_{\mathrm{ij}}$ components, with a standard uncertainty of $0.04 \AA^{2}$. Two severely disordered solvent THF molecules in the unit cell were removed by the SQUEEZE routine in PLATON. In 2, one of two THF molecules coordinated to potassium cation was found to be disordered. The disorder was modeled with two orientations having relative occupancies of 0.65:0.35 for the two parts. The geometries of the disordered parts were restrained to be similar. The anisotropic displacement parameters of the disordered molecules in the direction of the

bonds were restrained to be equal with a standard uncertainty of $0.01 \AA^{2}$. They were also restrained to have the same $\mathrm{U}_{\mathrm{ij}}$ components, with a standard uncertainty of $0.04 \AA^{2}$. The severely disordered solvent THF molecules were removed by the SQUEEZE routine in PLATON. ${ }^{5}$ In $\mathbf{3}$, the whole molecule was found to be disordered. The disorder was modeled with two orientations having relative occupancies of 0.50:0.50 for the two parts. The geometries of the disordered parts were restrained to be similar. The anisotropic displacement parameters of the disordered molecules in the direction of the bonds were restrained to be equal with a standard uncertainty of $0.01 \AA^{2}$. They were also restrained to have the same $\mathrm{U}_{\mathrm{ij}}$ components, with a standard uncertainty of $0.04 \AA^{2}$. Two severely disordered solvent THF molecules in the unit cell were removed by the SQUEEZE routine in PLATON. 
Table S1. Crystallographic data for 1, 2, and 3.

\begin{tabular}{|c|c|c|c|}
\hline Parameter & 1 & 2 & 3 \\
\hline Empirical formula & $\mathrm{C}_{88} \mathrm{H}_{116} \mathrm{KO}_{8} \mathrm{Si}_{2}$ & $\mathrm{C}_{108} \mathrm{H}_{156} \mathrm{~K}_{2} \mathrm{O}_{20} \mathrm{Si}_{2}$ & $\mathrm{C}_{88} \mathrm{H}_{120} \mathrm{~K}_{2} \mathrm{O}_{8} \mathrm{Si}_{2}$ \\
\hline$M_{\mathrm{r}}$ & 1397.09 & 1844.71 & 1440.22 \\
\hline Crystal system & Triclinic & Monoclinic & Triclinic \\
\hline Space group & $P-1$ & $P 2_{1} / c$ & $P-1$ \\
\hline$a(\AA)$ & $11.5995(3)$ & $14.2971(6)$ & $9.1690(4)$ \\
\hline$b(\AA)$ & $12.1738(3)$ & $31.7860(15)$ & $15.4719(7)$ \\
\hline$c(\AA)$ & $17.0652(4)$ & $13.0756(6)$ & $17.6353(7)$ \\
\hline$\alpha\left(^{\circ}\right)$ & $78.478(2)$ & 90 & $66.240(2)$ \\
\hline$\beta\left({ }^{\circ}\right)$ & $82.510(2)$ & $94.512(3)$ & $84.584(1)$ \\
\hline$\gamma\left(\left(^{\circ}\right)\right.$ & $72.257(1)$ & 90 & $81.803(2)$ \\
\hline$V\left(\AA^{3}\right)$ & $2242.66(10)$ & $5923.8(5)$ & $2264.45(17)$ \\
\hline$Z$ & 1 & 2 & 1 \\
\hline$\rho_{\text {calcd }}\left[\mathrm{g} \cdot \mathrm{cm}^{3}\right]$ & 1.034 & 1.034 & 1.056 \\
\hline$\mu\left[\mathrm{mm}^{-1}\right]$ & 1.15 & 1.33 & 1.55 \\
\hline $2 \theta$-range $[\mathrm{deg}]$ & $5.3-147.5$ & $6.2-149.2$ & $5.5-150.0$ \\
\hline Reflections collected & 38215 & 196398 & 51467 \\
\hline no. unique data, $R_{\text {int }}$ & $8603,0.062$ & $12111,0.096$ & $9027,0.046$ \\
\hline no. obsd data $[I \geq 2 \sigma(I)]$ & 6086 & 9254 & 6705 \\
\hline$R_{1}^{[a]}$ & 0.062 & 0.076 & 0.059 \\
\hline$w R_{2}^{[\mathrm{b}]}$ & 0.177 & 0.199 & 0.151 \\
\hline Data/restraints/parameters & $8603 / 150 / 517$ & $12111 / 151 / 632$ & $9027 / 1528 / 856$ \\
\hline Quality-of-fit ${ }^{[c]}$ & 1.08 & 1.09 & 1.04 \\
\hline peak/hole $\left[\mathrm{e} \cdot \AA^{-3}\right]$ & $0.58 /-0.62$ & $0.50 /-0.46$ & $0.33 /-0.25$ \\
\hline
\end{tabular}

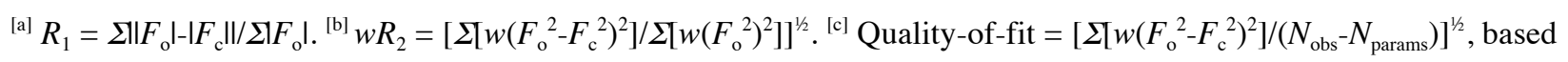
on all data. 


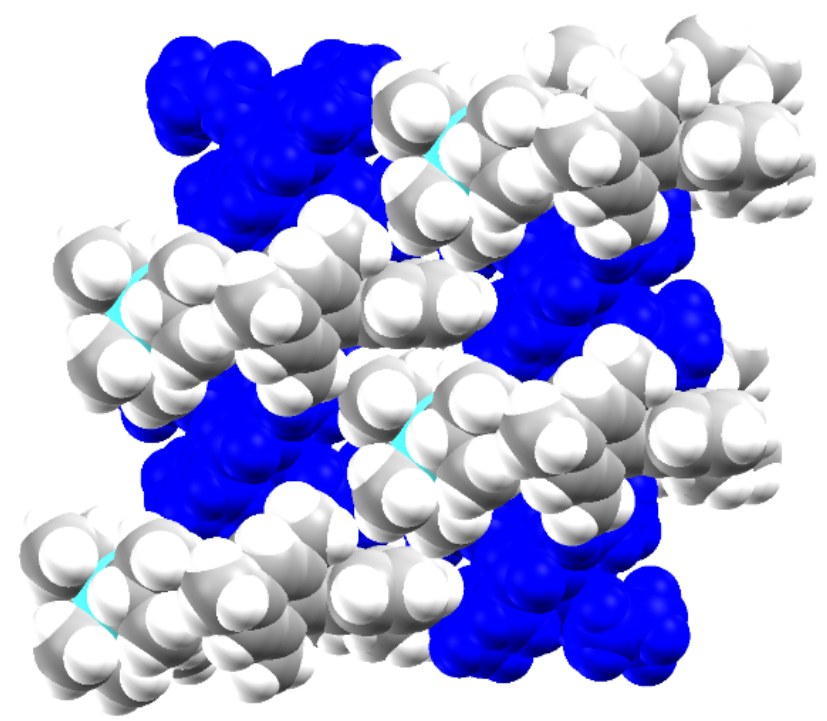

Figure S5. Solid-state packing of $\mathbf{1}$, space-filling model. The $\left[\left\{\mathrm{K}(18 \text {-crown-6)(THF })_{2}\right\}\right]^{+}$cations are shown in dark blue.

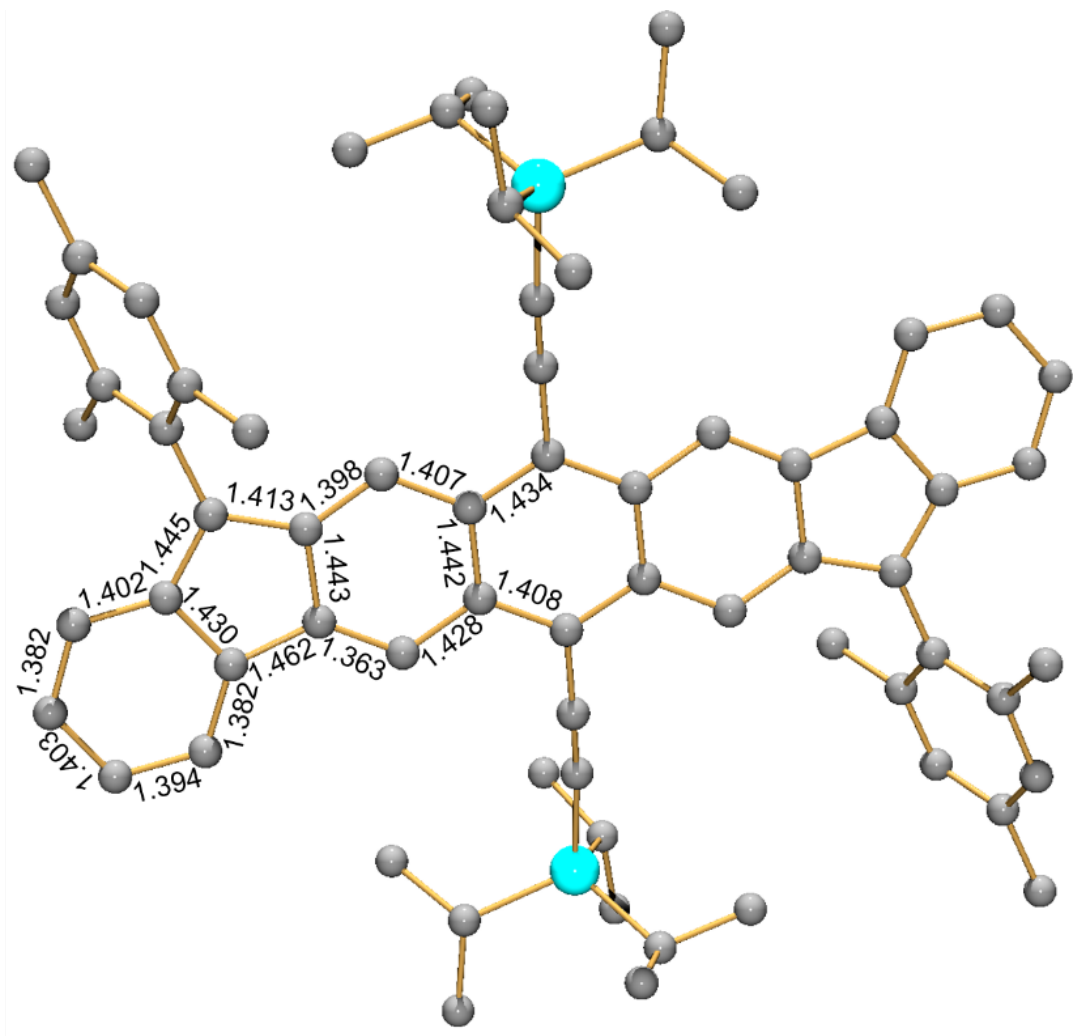

Figure S6. Key bond distances $(\AA)$ in the core of $\left[\left\{\mathrm{K}(18 \text {-crown-6)(THF })_{2}\right\} \mathrm{DIAn}^{0^{-}}\right](\mathbf{1})$. 


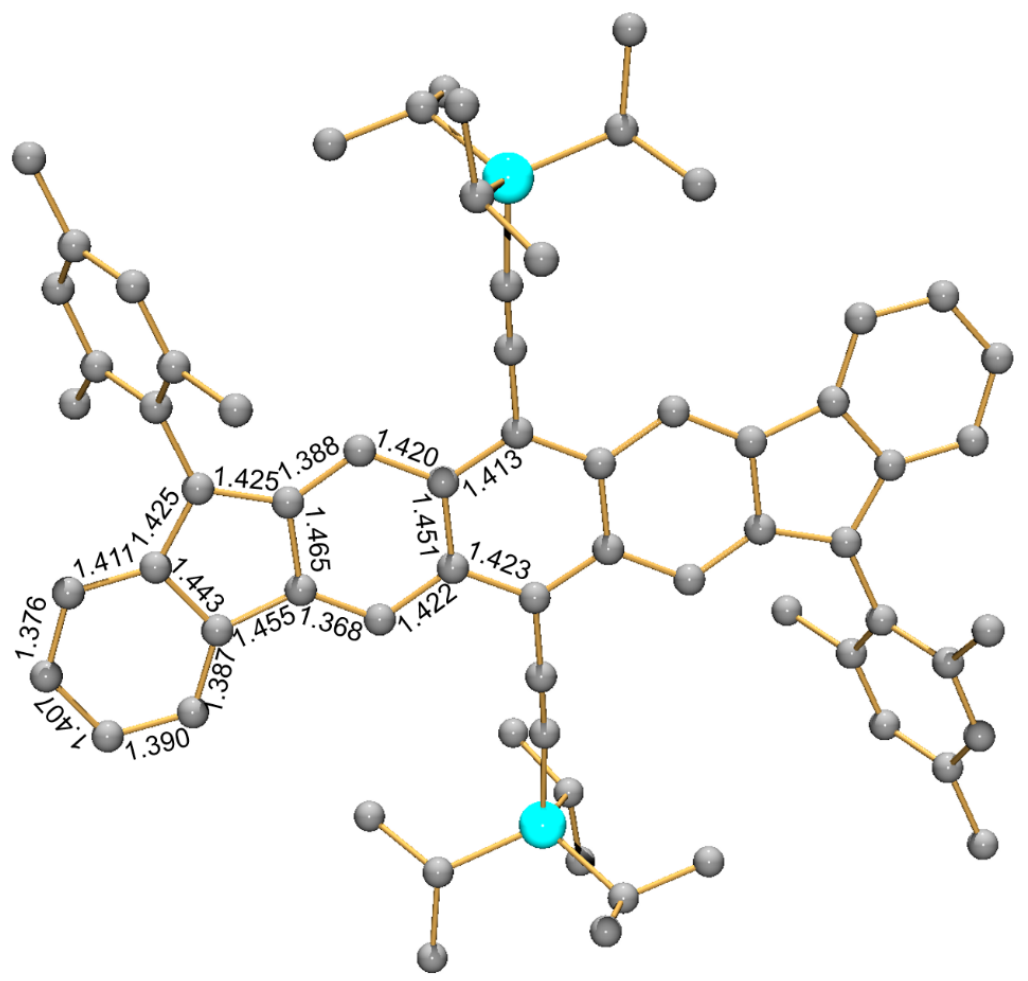

Figure S7. Key bond distances $(\AA)$ in the core of $\left[\left\{\mathrm{K}(18-\mathrm{crown}-6)(\mathrm{THF})_{2}\right\}_{2} \mathrm{DIAn}^{2-}\right](2)$.

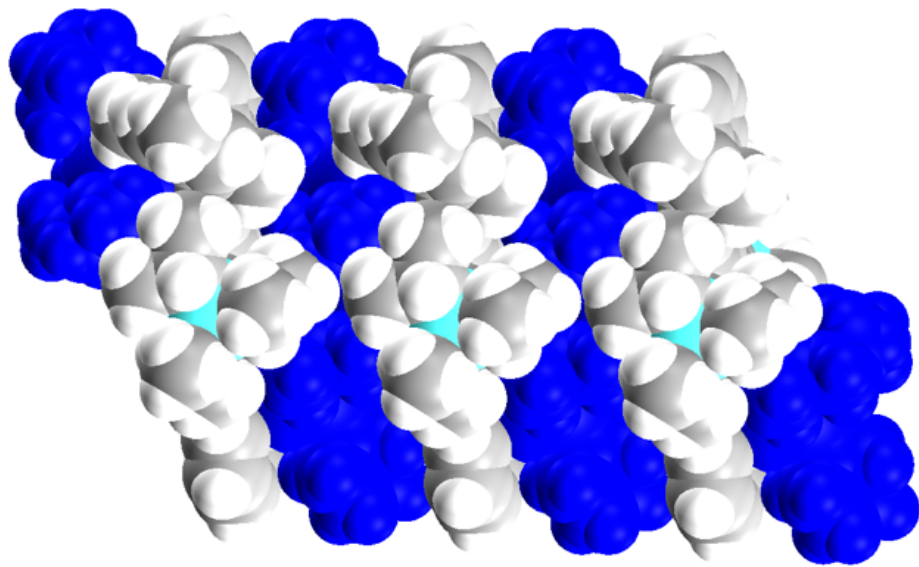

Figure S8. Solid-state packing of $\mathbf{3}$. The $[\{\mathrm{K}(\text { diglyme })(\mathrm{THF})\}]^{+}$cations are shown in dark blue. 


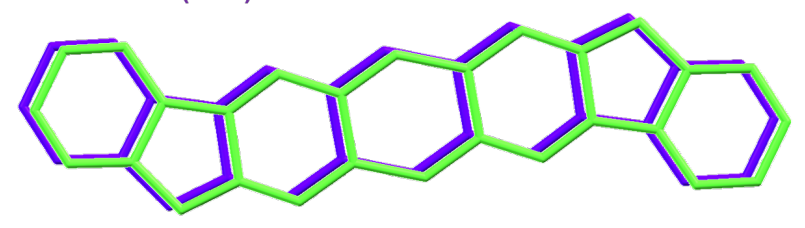

b

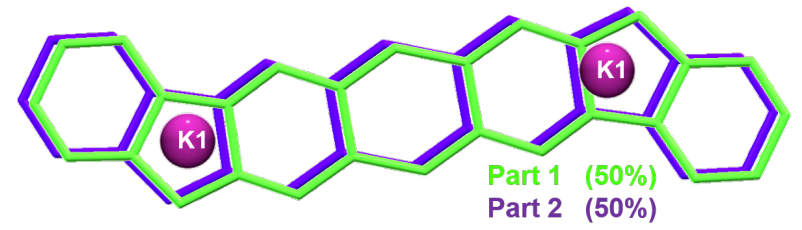

C

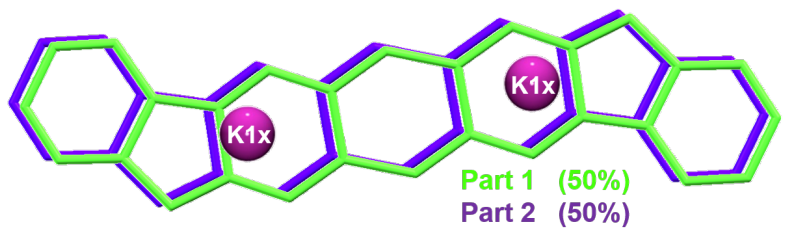

Figure S9. The anion and $\mathrm{K}$ ion are disordered over two orientations, which were resolved as part 1 and part 2. Disorder of the core of $\mathrm{C}_{68} \mathrm{H}_{76} \mathrm{Si}_{2}{ }^{2-}$ in 3 (a), and binding of $\mathrm{K}$ ions ( $\mathrm{b}$ and $\mathrm{c}$ ).

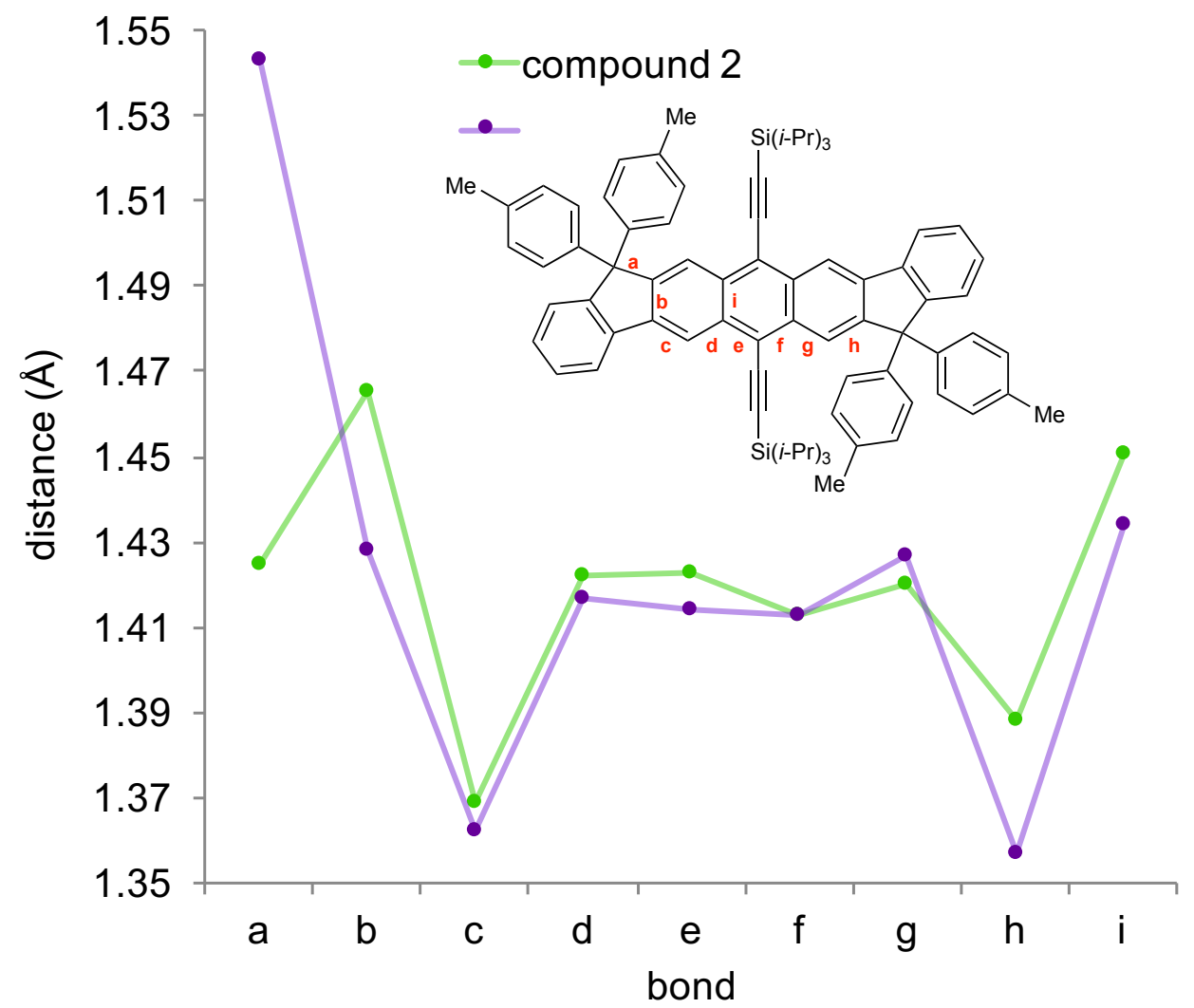

Figure S10. Comparison of the solid-state structures of the non-contact ion pair DIAn ${ }^{2-}$ (compound 2, green) and a tetrakis- $p$-tolyl DIAn derivative (violet). Bond lettering shown in red. 


\section{High-Pressure Raman Measurements}

High pressure experiments were conducted in a sapphire anvil cell (SAC) and moissanite anvil cell ${ }^{6,7}$ with a diameter culet of $360 \mu \mathrm{m}$ and a gold gasket, specified in each section. No pressure transmitting medium was used and diamond chips were placed as the pressure calibrant. Raman and photoluminescence measurements were performed using an air-cooled argon ion laser, a Spectra-Physics solid state laser, operating at $532 \mathrm{~nm}$. The device is equipped with a 10x Mitutoyo long working distance objective coupled to a 10x Navitar zoom system and focused onto the slit of an ISA HR460 monochromator with a grating of 600 grooves $\mathrm{mm}^{-1}$ and a liquid nitrogen cooled CCD detector (ISA CCD3000, 1024-256 pixels). Spectra were measured with a spectral resolution of about 2-3 $\mathrm{cm}^{-1}$ and calibrated with a standard neon emission lamp. The SAC is mounted on a xyz stage, which allows us to move the sample with an accuracy of $1 \mu \mathrm{m}$. The typical sampling area was about 1-2 $\mu \mathrm{m}$ in diameter.

Raman measurements were conducted at $633 \mathrm{~nm}$ with a Senterra Dispersive Raman Spectrophotometers from Bruker, which combines a dispersive Raman spectrophotometers with a confocal microscope in a single device. Detection is done through a high-sensitive CCD detector (charge couple device) with a Peltier-cooling to $-70{ }^{\circ} \mathrm{C}$ (thermoelectrically cooled). A grating of 1200 grooves

$\mathrm{mm}^{-1}$ provides a standard spectral resolution of $3 \mathrm{~cm}^{-1}$. Measurements were done with $5 \mathrm{~mW}$ power. The calibration wavelength is continuous throughout the process of the spectral registration. The method used calibrates the system automatically with unmatched wavelength precision and accuracy using a Neon light as reference. Raman scattering radiation is collected in back-scattering configuration spatial resolution of $0.5 \mu \mathrm{m}$, and spot size of about $3 \mu \mathrm{m}$. 


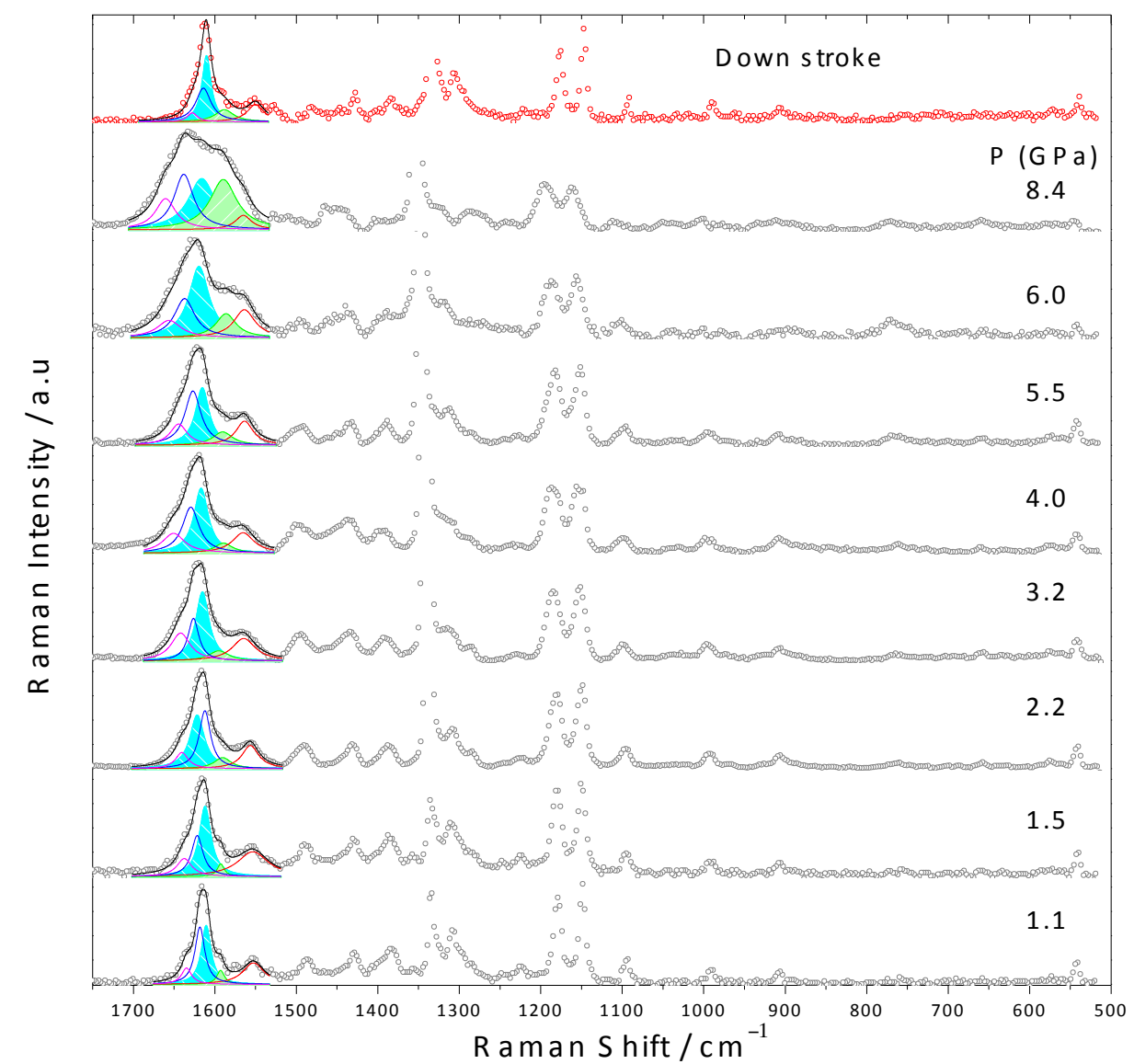

Figure S11. Raman spectrum at selected pressures measured with a laser of $532 \mathrm{~nm}$ in the $1750-500 \mathrm{~cm}^{-1}$ region. the background has been subtracted to obtain better visibility.
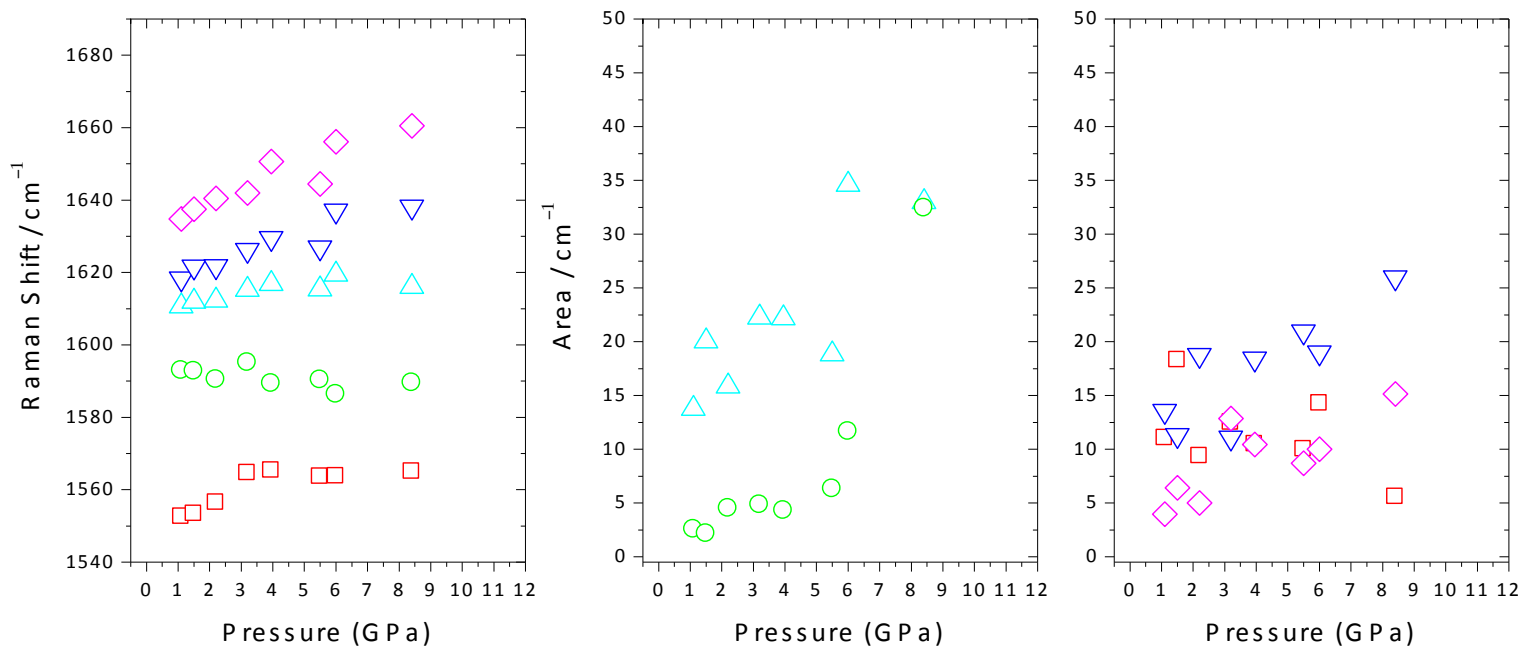

Figure S12. (left) Raman shift pressure evolution of the contributions fitted as in previous figure from experimental Raman spectra taken with $532 \mathrm{~nm}$ as excitation line; each color corresponds to the fitted contribution. (middle) Area obtained for the 1610 and $1589 \mathrm{~cm}^{-1}$ contributions (cyan and green). (right) Area obtained for the 1637, 1620 and $1555 \mathrm{~cm}^{-1}$ contributions (pink, dark blue and red, respectively). 


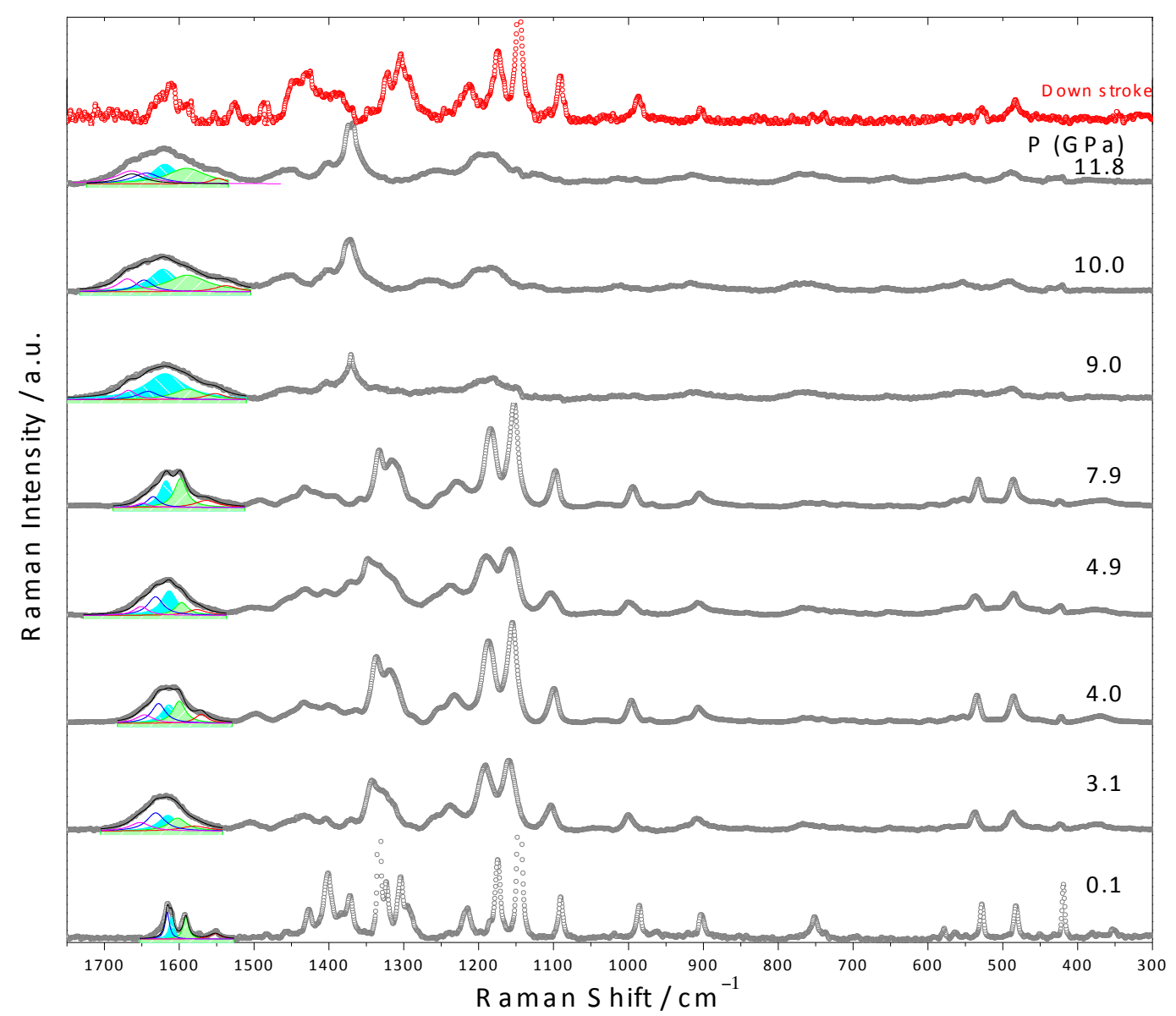

Figure S13. Raman spectrum at selected pressures measured with a laser of $633 \mathrm{~nm}$ in the $1750-300 \mathrm{~cm}^{-1}$ region. The background has been subtracted to obtain better visibility.
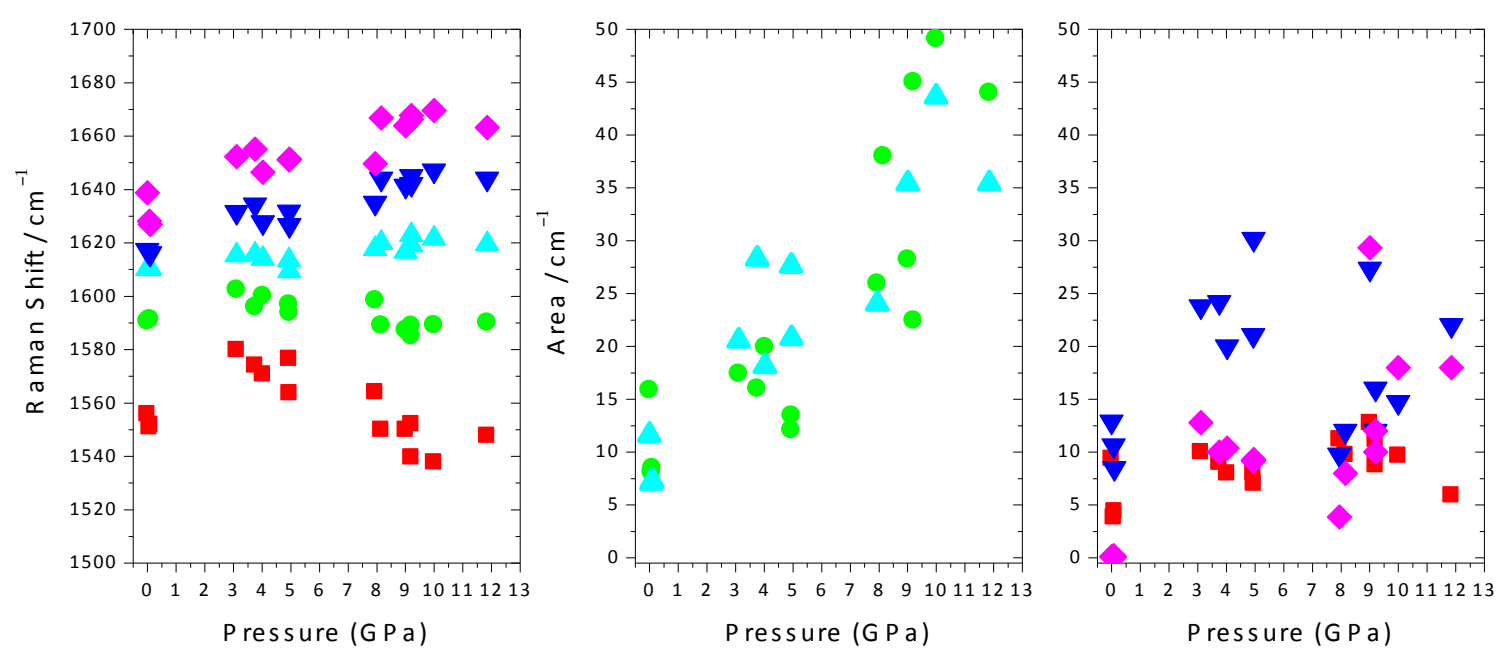

Figure S14. (left) Raman shift pressure evolution of the contributions fitted as in previous figure from experimental Raman spectra taken with $633 \mathrm{~nm}$ as excitation line, each color corresponds to the fitted contribution. (middle) Area obtained for the 1610 and $1589 \mathrm{~cm}^{-1}$ contributions (cyan and green) (right) Area obtained for the 1637, 1620 and $1555 \mathrm{~cm}^{-1}$ contributions (pink, dark blue, and red, respectively) . 
In both spectra sets $(532 \mathrm{~nm}$ and $633 \mathrm{~nm})$, it is seen how with increasing pressure induces the intensity growth of the 1610 and $1591 \mathrm{~cm}^{-1}$ bands, which could indicate that pressure leads towards the aromatization of the core.
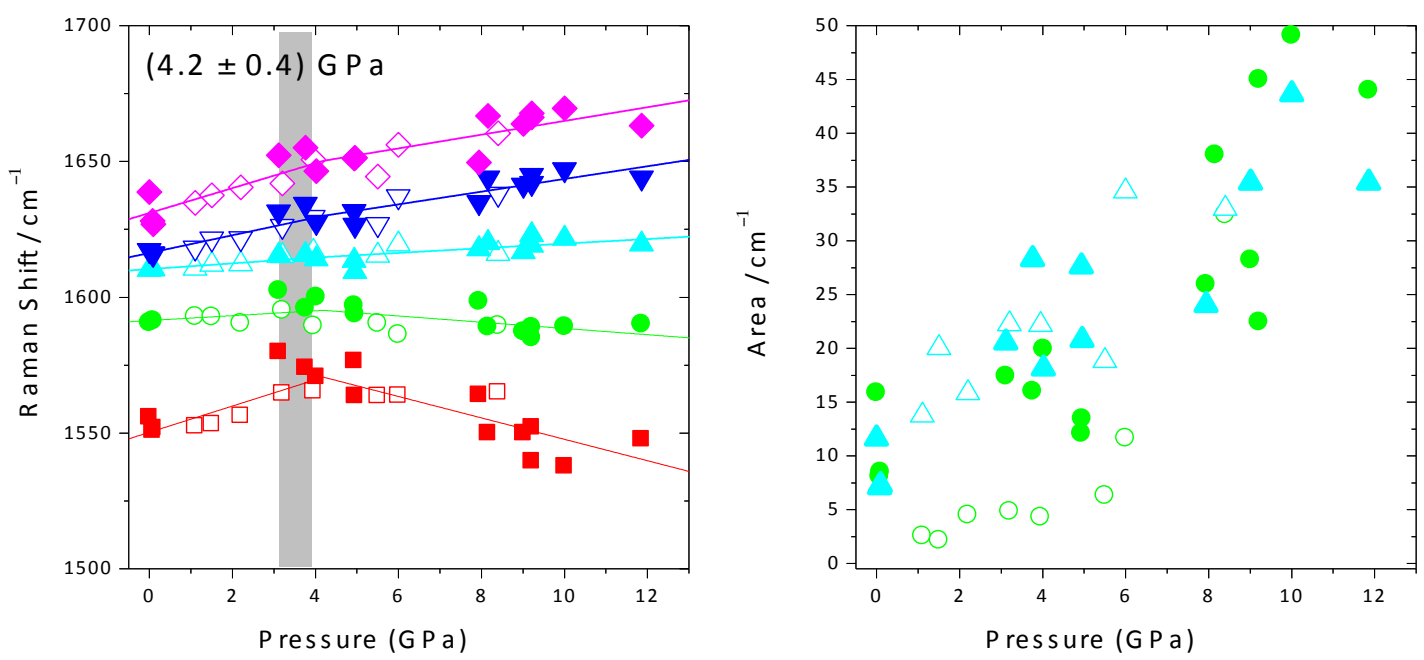

Figure S15. (left) Raman shift pressure evolution of the contributions fitted as in previous figure from experimental Raman spectra taken with 633 and $532 \mathrm{~nm}$, filled and empty symbols respectively; each color corresponds to the fitted contribution. (right) Area obtained for the 1610 and $1589 \mathrm{~cm}^{-1}$ contributions (cyan and green)

Table S2. Experimental interceptions and pressure coefficients of the Raman bands

\begin{tabular}{|l|l|l|l|l|l|}
\hline$\omega_{0}\left(\mathrm{~cm}^{-1}\right)$ & 1637 & 1616 & 1610 & 1591 & 1551 \\
\hline $\mathrm{S}_{1}\left(\mathrm{~cm}^{-1} / \mathrm{GPa}\right)$ & $4.6 \pm 0.7$ & $3.3 \pm 0.7$ & $1.1 \pm 0.7$ & $0.9 \pm 0.7$ & $4.8 \pm 0.9$ \\
\hline $\mathrm{S}_{2}\left(\mathrm{~cm}^{-1} / \mathrm{GPa}\right)$ & $2.5 \pm 0.5$ & $2.3 \pm 0.5$ & $0.8 \pm 0.5$ & $-1.1 \pm 0.5$ & $-3.9 \pm 0.6$ \\
\hline
\end{tabular}

The observed shifts of all the contributions with pressure show a two regime stage where the different Raman bands shift with different slope with increasing pressure. We have conducted a fitting of all the bands with a shared pressure transition, with the data obtained from the fittings of the $633 \mathrm{~nm}$ and $532 \mathrm{~nm}$ spectra. We obtain a pressure transition of $(4.2 \pm 0.4) \mathrm{GPa}$, from this the slopes of any contribution are smaller than in the previous stage. In the literature of PAHs changes in slopes are related with phase transition within the crystal phase group ${ }^{8-10}$ however, those changes in slopes described for anthracene for instance are softer than the ones obtained for DIAn. Additionally, it should be mentioned that the 1600 and $1591 \mathrm{~cm}^{-1}$ show slopes below those observed for other PAHs, and lower than the other DIAn contribution. On the other hand, at pressures above pressure transition not only the slopes of these two contributions, 1600 and $1591 \mathrm{~cm}^{-1}$ bands are lower, but in the case of the $1591 \mathrm{~cm}^{-1}$ this is negative. These results corroborate that at pressures above 4.2 GPa the aromatic core is favored, explaining the intensity increase of the corresponding features and the lower slopes of the $\mathrm{C}-\mathrm{C}$ contributions. 


\section{Computational Details}

\section{Geometry Optimization}

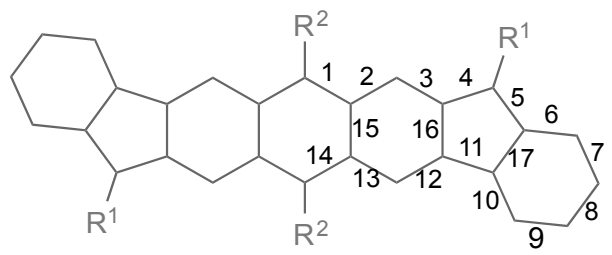

Figure S16. Bond numbering scheme for geometry optimization.

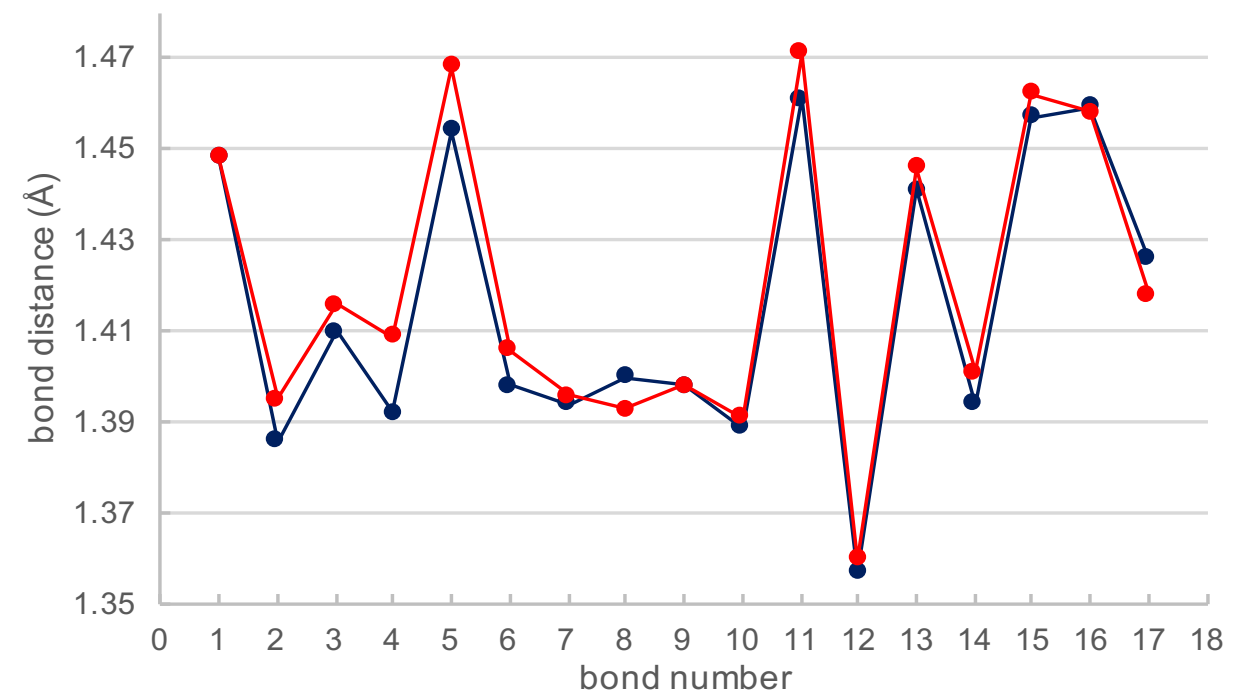

Figure S17. Bond distances from the solid-state structure of DIAn (navy) and DFT optimized geometry (red) of model DIAn. RMS deviation $=0.008 \AA$.

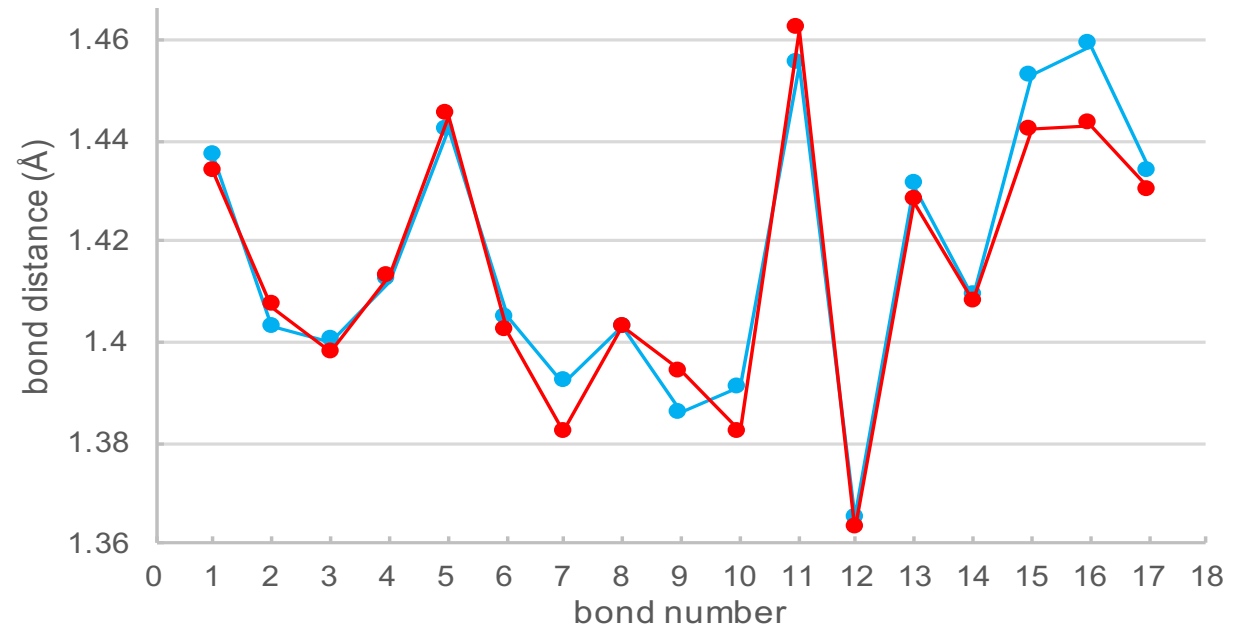

Figure S18. Bond distances from the solid-state structure of radical anion 1 (red) and DFT optimized geometry (blue) of model DIAn- ${ }^{\circ}$. RMS deviation $=0.007 \AA ̊$. 


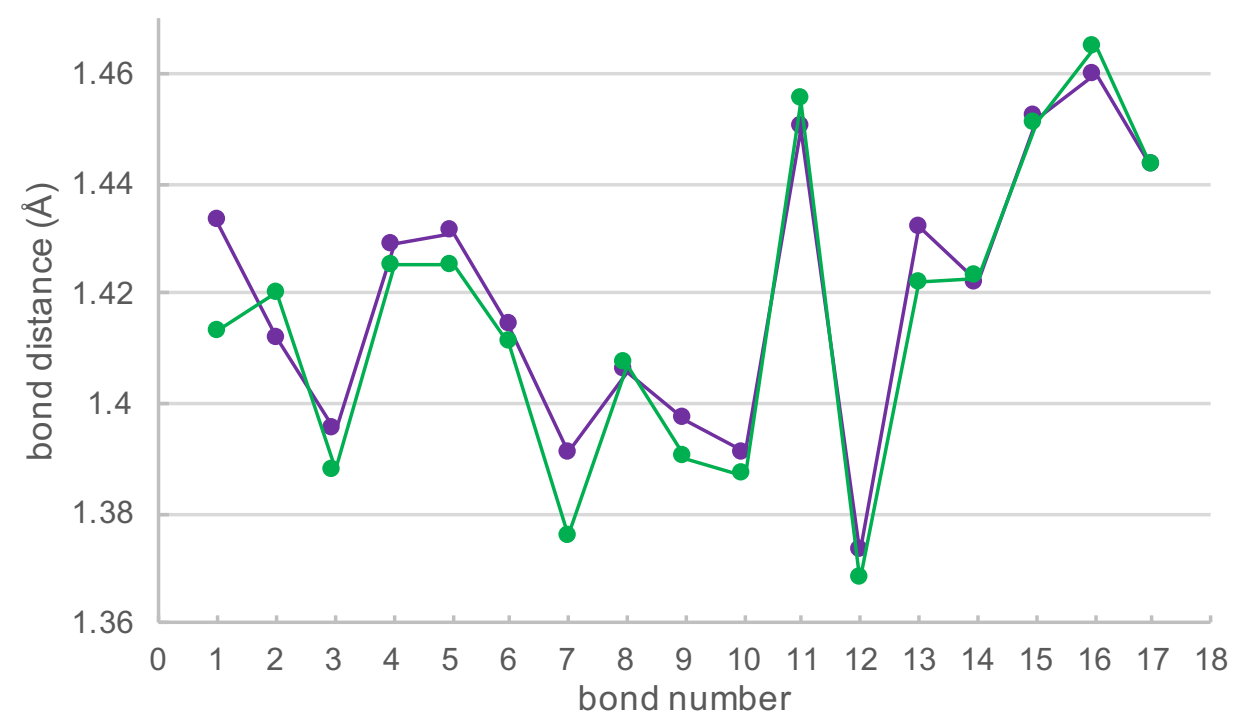

Figure S19. Bond distances from the solid-state structure of dianion 2 (green) and DFT optimized geometry (violet) of model DIAn ${ }^{2-}$. RMS deviation $=0.008 \AA$.

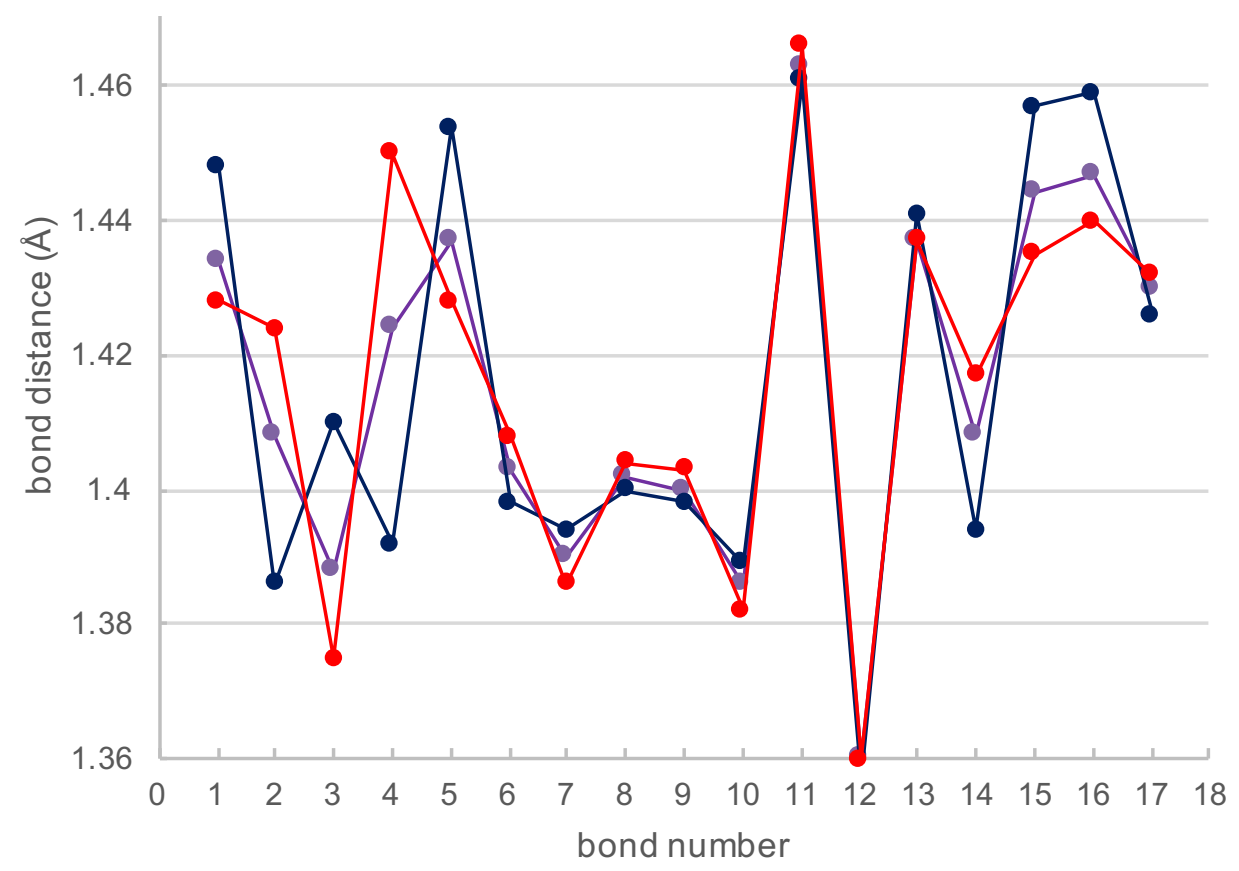

Figure S20. Bond distances from the DFT optimized geometries of the computationally simplified models for DIAn (navy), DIAn ${ }^{*+}$ (purple), and DIAn $^{2+}$ (red). 


\section{Cartesian Coordinates}

Table S3. Optimized geometry of DIAn calculated at RB3LYP/6-311G* level of theory .

Zero-point correction $=0.837187$ (Hartree/Particle)

Thermal correction to Energy $=0.893461$

Thermal correction to Enthalpy $=0.894405$

Thermal correction to Gibbs Free Energy $=0.738425$

Sum of electronic and zero-point Energies $=-2665.313495$

Sum of electronic and thermal Energies $=-2665.257222$

Sum of electronic and thermal Enthalpies $=-2665.256277$

Sum of electronic and thermal Free Energies $=-2665.412257$

NImag $=0$

\begin{tabular}{|c|c|c|c|}
\hline & $x$ & $y$ & $z$ \\
\hline $\mathrm{C}$ & 1.198133 & 0.785288 & 0.100175 \\
\hline $\mathrm{C}$ & 2.377084 & 1.608252 & 0.198490 \\
\hline $\mathrm{H}$ & 3.341749 & 1.114431 & 0.231178 \\
\hline $\mathrm{C}$ & 2.274909 & 2.960386 & 0.247085 \\
\hline $\mathrm{C}$ & 3.269581 & 4.026014 & 0.343470 \\
\hline $\mathrm{C}$ & 4.655646 & 4.012597 & 0.424720 \\
\hline $\mathrm{H}$ & 5.206309 & 3.076690 & 0.420415 \\
\hline $\mathrm{C}$ & 5.340055 & 5.228112 & 0.514378 \\
\hline $\mathrm{H}$ & 6.423301 & 5.231145 & 0.578123 \\
\hline $\mathrm{C}$ & 4.643307 & 6.441923 & 0.524961 \\
\hline $\mathrm{H}$ & 5.195506 & 7.373549 & 0.597842 \\
\hline $\mathrm{C}$ & 3.251935 & 6.470616 & 0.445213 \\
\hline $\mathrm{H}$ & 2.716862 & 7.414896 & 0.458185 \\
\hline $\mathrm{C}$ & 2.556757 & 5.261053 & 0.351022 \\
\hline $\mathrm{C}$ & 1.131548 & 4.989137 & 0.262524 \\
\hline $\mathrm{C}$ & 0.968246 & 3.607671 & 0.200980 \\
\hline $\mathrm{C}$ & -0.193186 & 2.814132 & 0.099815 \\
\hline $\mathrm{H}$ & -1.166251 & 3.289455 & 0.057640 \\
\hline $\mathrm{C}$ & -0.106375 & 1.431300 & 0.050442 \\
\hline $\mathrm{C}$ & 0.056982 & 6.012734 & 0.245491 \\
\hline $\mathrm{C}$ & -0.635760 & 6.323854 & 1.434364 \\
\hline $\mathrm{C}$ & -1.644837 & 7.290158 & 1.396094 \\
\hline $\mathrm{H}$ & -2.177079 & 7.537558 & 2.309971 \\
\hline $\mathrm{C}$ & -1.968011 & 7.940093 & 0.210239 \\
\hline $\mathrm{C}$ & -1.280041 & 7.630677 & -0.957774 \\
\hline $\mathrm{H}$ & -1.532881 & 8.138062 & -1.884217 \\
\hline $\mathrm{C}$ & -0.263850 & 6.671754 & -0.960196 \\
\hline $\mathrm{H}$ & 0.770590 & 5.698546 & 2.960723 \\
\hline $\mathrm{H}$ & -2.753408 & 8.689543 & 0.196981 \\
\hline $\mathrm{Si}$ & -5.256027 & 2.694990 & -0.203814 \\
\hline
\end{tabular}




\begin{tabular}{|c|c|c|c|}
\hline $\mathrm{C}$ & -1.291355 & 0.604916 & -0.051136 \\
\hline $\mathrm{C}$ & -2.561012 & 1.242307 & -0.099653 \\
\hline $\mathrm{C}$ & -3.635816 & 1.811868 & -0.140546 \\
\hline $\mathrm{C}$ & -6.631963 & 1.406348 & -0.227186 \\
\hline $\mathrm{H}$ & -6.553065 & 0.750927 & -1.099179 \\
\hline $\mathrm{H}$ & -6.605790 & 0.774995 & 0.665440 \\
\hline $\mathrm{H}$ & -7.616211 & 1.884197 & -0.263328 \\
\hline $\mathrm{C}$ & -5.398866 & 3.788052 & 1.324331 \\
\hline $\mathrm{H}$ & -4.599901 & 4.533854 & 1.358170 \\
\hline $\mathrm{H}$ & -5.342161 & 3.200950 & 2.245068 \\
\hline $\mathrm{H}$ & -6.352819 & 4.325319 & 1.332541 \\
\hline $\mathrm{C}$ & -5.303314 & 3.741656 & -1.769933 \\
\hline $\mathrm{H}$ & -6.254284 & 4.277794 & -1.853243 \\
\hline $\mathrm{H}$ & -5.190202 & 3.127358 & -2.667407 \\
\hline $\mathrm{H}$ & -4.502863 & 4.486529 & -1.776940 \\
\hline $\mathrm{C}$ & -0.297732 & 5.636431 & 2.736790 \\
\hline $\mathrm{H}$ & -0.551688 & 4.572764 & 2.711625 \\
\hline $\mathrm{H}$ & -0.842289 & 6.087606 & 3.568716 \\
\hline $\mathrm{C}$ & 0.461453 & 6.340971 & -2.243447 \\
\hline $\mathrm{H}$ & 0.069186 & 6.927300 & -3.076752 \\
\hline $\mathrm{H}$ & 0.359884 & 5.282812 & -2.500593 \\
\hline $\mathrm{H}$ & 1.533600 & 6.543136 & -2.168014 \\
\hline $\mathrm{C}$ & -1.198133 & -0.785288 & -0.100175 \\
\hline $\mathrm{C}$ & -2.377084 & -1.608252 & -0.198490 \\
\hline $\mathrm{H}$ & -3.341749 & -1.114431 & -0.231178 \\
\hline $\mathrm{C}$ & -2.274909 & -2.960386 & -0.247085 \\
\hline $\mathrm{C}$ & -3.269581 & -4.026014 & -0.343470 \\
\hline $\mathrm{C}$ & -4.655646 & -4.012597 & -0.424720 \\
\hline $\mathrm{H}$ & -5.206309 & -3.076690 & -0.420415 \\
\hline $\mathrm{C}$ & -5.340055 & -5.228112 & -0.514378 \\
\hline $\mathrm{H}$ & -6.423301 & -5.231145 & -0.578123 \\
\hline $\mathrm{C}$ & -4.643307 & -6.441923 & -0.524961 \\
\hline $\mathrm{H}$ & -5.195506 & -7.373549 & -0.597842 \\
\hline $\mathrm{C}$ & -3.251935 & -6.470616 & -0.445213 \\
\hline $\mathrm{H}$ & -2.716862 & -7.414896 & -0.458185 \\
\hline $\mathrm{C}$ & -2.556757 & -5.261053 & -0.351022 \\
\hline $\mathrm{C}$ & -1.131548 & -4.989137 & -0.262524 \\
\hline $\mathrm{C}$ & -0.968246 & -3.607671 & -0.200980 \\
\hline $\mathrm{C}$ & 0.193186 & -2.814132 & -0.099815 \\
\hline $\mathrm{H}$ & 1.166251 & -3.289455 & -0.057640 \\
\hline $\mathrm{C}$ & 0.106375 & -1.431300 & -0.050442 \\
\hline $\mathrm{C}$ & -0.056982 & -6.012734 & -0.245491 \\
\hline $\mathrm{C}$ & 0.635760 & -6.323854 & -1.434364 \\
\hline $\mathrm{C}$ & 1.644837 & -7.290158 & -1.396094 \\
\hline $\mathrm{H}$ & 2.177079 & -7.537558 & -2.309971 \\
\hline
\end{tabular}




\begin{tabular}{rrrr}
\hline $\mathrm{C}$ & 1.968011 & -7.940093 & -0.210239 \\
$\mathrm{C}$ & 1.280041 & -7.630677 & 0.957774 \\
$\mathrm{H}$ & 1.532881 & -8.138062 & 1.884217 \\
$\mathrm{C}$ & 0.263850 & -6.671754 & 0.960196 \\
$\mathrm{H}$ & -0.770590 & -5.698546 & -2.960723 \\
$\mathrm{H}$ & 2.753408 & -8.689543 & -0.196981 \\
$\mathrm{Si}$ & 5.256027 & -2.694990 & 0.203814 \\
$\mathrm{C}$ & 1.291355 & -0.604916 & 0.051136 \\
$\mathrm{C}$ & 2.561012 & -1.242307 & 0.099653 \\
$\mathrm{C}$ & 3.635816 & -1.811868 & 0.140546 \\
$\mathrm{C}$ & 6.631963 & -1.406348 & 0.227186 \\
$\mathrm{H}$ & 6.553065 & -0.750927 & 1.099179 \\
$\mathrm{H}$ & 6.605790 & -0.774995 & -0.665440 \\
$\mathrm{H}$ & 7.616211 & -1.884197 & 0.263328 \\
$\mathrm{C}$ & 5.398866 & -3.788052 & -1.324331 \\
$\mathrm{H}$ & 4.599901 & -4.533854 & -1.358170 \\
$\mathrm{H}$ & 5.342161 & -3.200950 & -2.245068 \\
$\mathrm{H}$ & 6.352819 & -4.325319 & -1.332541 \\
$\mathrm{C}$ & 5.303314 & -3.741656 & 1.769933 \\
$\mathrm{H}$ & 6.254284 & -4.277794 & 1.853243 \\
$\mathrm{H}$ & 5.190202 & -3.127358 & 2.667407 \\
$\mathrm{H}$ & 4.502863 & -4.486529 & 1.776940 \\
$\mathrm{C}$ & 0.297732 & -5.636431 & -2.736790 \\
$\mathrm{H}$ & 0.551688 & -4.572764 & -2.711625 \\
$\mathrm{H}$ & 0.842289 & -6.087606 & -3.568716 \\
$\mathrm{C}$ & -0.461453 & -6.340971 & 2.243447 \\
$\mathrm{H}$ & -0.069186 & -6.927300 & 3.076752 \\
$\mathrm{H}$ & -0.359884 & -5.282812 & 2.500593 \\
$\mathrm{H}$ & -1.533600 & -6.543136 & 2.168014 \\
\hline & & & \\
\hline
\end{tabular}


Table S4 . Optimized geometry of DIAn*+ calculated at UB3LYP/6-311G* level of theory.

Zero-point correction $=0.837848$ (Hartree/Particle $)$

Thermal correction to Energy $=0.894313$

Thermal correction to Enthalpy $=0.895257$

Thermal correction to Gibbs Free Energy $=0.737820$

Sum of electronic and zero-point Energies $=-2665.109046$

Sum of electronic and thermal Energies $=-2665.052581$

Sum of electronic and thermal Enthalpies $=-2665.051637$

Sum of electronic and thermal Free Energies $=-2665.209074$

NImag $=0$

\begin{tabular}{lrlc}
\hline & $x$ & $y$ & $z$ \\
\hline $\mathrm{C}$ & 1.197885 & 0.780993 & 0.099923 \\
$\mathrm{C}$ & 2.380549 & 1.591726 & 0.196157 \\
$\mathrm{H}$ & 3.342093 & 1.092756 & 0.223776 \\
$\mathrm{C}$ & 2.280379 & 2.947053 & 0.247011 \\
$\mathrm{C}$ & 3.272937 & 4.018649 & 0.336140 \\
$\mathrm{C}$ & 4.656423 & 4.009612 & 0.414512 \\
$\mathrm{H}$ & 5.215666 & 3.079965 & 0.413455 \\
$\mathrm{C}$ & 5.331387 & 5.233019 & 0.498645 \\
$\mathrm{H}$ & 6.414213 & 5.239635 & 0.560414 \\
$\mathrm{C}$ & 4.636704 & 6.451030 & 0.508379 \\
$\mathrm{H}$ & 5.191044 & 7.380139 & 0.578539 \\
$\mathrm{C}$ & 3.249404 & 6.476561 & 0.432018 \\
$\mathrm{H}$ & 2.707552 & 7.416052 & 0.444651 \\
$\mathrm{C}$ & 2.559976 & 5.257790 & 0.341157 \\
$\mathrm{C}$ & 1.149261 & 4.997109 & 0.262969 \\
$\mathrm{C}$ & 0.981642 & 3.584513 & 0.205617 \\
$\mathrm{C}$ & -0.174742 & 2.824148 & 0.104623 \\
$\mathrm{H}$ & -1.144850 & 3.305204 & 0.064164 \\
$\mathrm{C}$ & -0.096291 & 1.418884 & 0.051731 \\
$\mathrm{C}$ & 0.069781 & 6.009360 & 0.246476 \\
$\mathrm{C}$ & -0.629351 & 6.299499 & 1.437805 \\
$\mathrm{C}$ & -1.641479 & 7.261275 & 1.398512 \\
$\mathrm{H}$ & -2.177032 & 7.503096 & 2.311154 \\
$\mathrm{C}$ & -1.961364 & 7.916669 & 0.214715 \\
$\mathrm{C}$ & -1.266715 & 7.621361 & -0.952859 \\
$\mathrm{H}$ & -1.518704 & 8.134655 & -1.875447 \\
$\mathrm{C}$ & -0.241898 & 6.672648 & -0.959954 \\
$\mathrm{H}$ & 0.774590 & 5.712802 & 2.985608 \\
$\mathrm{H}$ & -2.749361 & 8.662398 & 0.203265 \\
$\mathrm{Si}$ & -5.273663 & 2.698146 & -0.208275 \\
$\mathrm{C}$ & -1.285658 & 0.623368 & -0.049169 \\
$\mathrm{C}$ & -2.549887 & 1.264750 & -0.097724 \\
\hline & & &
\end{tabular}




\begin{tabular}{|c|c|c|c|}
\hline $\mathrm{C}$ & -3.628647 & 1.827020 & -0.140434 \\
\hline $\mathrm{C}$ & -6.614509 & 1.378175 & -0.230581 \\
\hline $\mathrm{H}$ & -6.525372 & 0.725865 & -1.103819 \\
\hline $\mathrm{H}$ & -6.579002 & 0.749904 & 0.663779 \\
\hline $\mathrm{H}$ & -7.607480 & 1.836660 & -0.267196 \\
\hline $\mathrm{C}$ & -5.405580 & 3.781180 & 1.323952 \\
\hline $\mathrm{H}$ & -4.614452 & 4.535309 & 1.354439 \\
\hline $\mathrm{H}$ & -5.341301 & 3.193474 & 2.243546 \\
\hline $\mathrm{H}$ & -6.363548 & 4.310312 & 1.338990 \\
\hline $\mathrm{C}$ & -5.303944 & 3.730684 & -1.780101 \\
\hline $\mathrm{H}$ & -6.258660 & 4.257367 & -1.875223 \\
\hline $\mathrm{H}$ & -5.179355 & 3.113706 & -2.673930 \\
\hline $\mathrm{H}$ & -4.512192 & 4.484654 & -1.783691 \\
\hline $\mathrm{C}$ & -0.286726 & 5.614030 & 2.740506 \\
\hline $\mathrm{H}$ & -0.507934 & 4.542979 & 2.711534 \\
\hline $\mathrm{H}$ & -0.855901 & 6.042560 & 3.566480 \\
\hline $\mathrm{C}$ & 0.493057 & 6.360676 & -2.242917 \\
\hline $\mathrm{H}$ & 0.079859 & 6.930046 & -3.076467 \\
\hline $\mathrm{H}$ & 0.426217 & 5.300008 & -2.502772 \\
\hline $\mathrm{H}$ & 1.557226 & 6.603986 & -2.175103 \\
\hline $\mathrm{C}$ & -1.197885 & -0.780993 & -0.099923 \\
\hline $\mathrm{C}$ & -2.380549 & -1.591726 & -0.196157 \\
\hline $\mathrm{H}$ & -3.342093 & -1.092756 & -0.223776 \\
\hline $\mathrm{C}$ & -2.280379 & -2.947053 & -0.247011 \\
\hline $\mathrm{C}$ & -3.272937 & -4.018649 & -0.336140 \\
\hline $\mathrm{C}$ & -4.656423 & -4.009612 & -0.414512 \\
\hline $\mathrm{H}$ & -5.215666 & -3.079965 & -0.413455 \\
\hline $\mathrm{C}$ & -5.331387 & -5.233019 & -0.498645 \\
\hline $\mathrm{H}$ & -6.414213 & -5.239635 & -0.560414 \\
\hline $\mathrm{C}$ & -4.636704 & -6.451030 & -0.508379 \\
\hline $\mathrm{H}$ & -5.191044 & -7.380139 & -0.578539 \\
\hline $\mathrm{C}$ & -3.249404 & -6.476561 & -0.432018 \\
\hline $\mathrm{H}$ & -2.707552 & -7.416052 & -0.444651 \\
\hline $\mathrm{C}$ & -2.559976 & -5.257790 & -0.341157 \\
\hline $\mathrm{C}$ & -1.149261 & -4.997109 & -0.262969 \\
\hline $\mathrm{C}$ & -0.981642 & -3.584513 & -0.205617 \\
\hline $\mathrm{C}$ & 0.174742 & -2.824148 & -0.104623 \\
\hline $\mathrm{H}$ & 1.144850 & -3.305204 & -0.064164 \\
\hline $\mathrm{C}$ & 0.096291 & -1.418884 & -0.051731 \\
\hline $\mathrm{C}$ & -0.069781 & -6.009360 & -0.246476 \\
\hline $\mathrm{C}$ & 0.629351 & -6.299499 & -1.437805 \\
\hline $\mathrm{C}$ & 1.641479 & -7.261275 & -1.398512 \\
\hline $\mathrm{H}$ & 2.177032 & -7.503096 & -2.311154 \\
\hline $\mathrm{C}$ & 1.961364 & -7.916669 & -0.214715 \\
\hline $\mathrm{C}$ & 1.266715 & -7.621361 & 0.952859 \\
\hline
\end{tabular}




\begin{tabular}{rrrr}
\hline $\mathrm{H}$ & 1.518704 & -8.134655 & 1.875447 \\
$\mathrm{C}$ & 0.241898 & -6.672648 & 0.959954 \\
$\mathrm{H}$ & -0.774590 & -5.712802 & -2.985608 \\
$\mathrm{H}$ & 2.749361 & -8.662398 & -0.203265 \\
$\mathrm{Si}$ & 5.273663 & -2.698146 & 0.208275 \\
$\mathrm{C}$ & 1.285658 & -0.623368 & 0.049169 \\
$\mathrm{C}$ & 2.549887 & -1.264750 & 0.097724 \\
$\mathrm{C}$ & 3.628647 & -1.827020 & 0.140434 \\
$\mathrm{C}$ & 6.614509 & -1.378175 & 0.230581 \\
$\mathrm{H}$ & 6.525372 & -0.725865 & 1.103819 \\
$\mathrm{H}$ & 6.579002 & -0.749904 & -0.663779 \\
$\mathrm{H}$ & 7.607480 & -1.836660 & 0.267196 \\
$\mathrm{C}$ & 5.405580 & -3.781180 & -1.323952 \\
$\mathrm{H}$ & 4.614452 & -4.535309 & -1.354439 \\
$\mathrm{H}$ & 5.341301 & -3.193474 & -2.243546 \\
$\mathrm{H}$ & 6.363548 & -4.310312 & -1.338990 \\
$\mathrm{C}$ & 5.303944 & -3.730684 & 1.780101 \\
$\mathrm{H}$ & 6.258660 & -4.257367 & 1.875223 \\
$\mathrm{H}$ & 5.179355 & -3.113706 & 2.673930 \\
$\mathrm{H}$ & 4.512192 & -4.484654 & 1.783691 \\
$\mathrm{C}$ & 0.286726 & -5.614030 & -2.740506 \\
$\mathrm{H}$ & 0.507934 & -4.542979 & -2.711534 \\
$\mathrm{H}$ & 0.855901 & -6.042560 & -3.566480 \\
$\mathrm{C}$ & -0.493057 & -6.360676 & 2.242917 \\
$\mathrm{H}$ & -0.079859 & -6.930046 & 3.076467 \\
$\mathrm{H}$ & -0.426217 & -5.300008 & 2.502772 \\
$\mathrm{H}$ & -1.557226 & -6.603986 & 2.175103 \\
\hline & & & \\
\hline
\end{tabular}


Table S5. Optimized geometry of DIAn'- calculated at UB3LYP/6-311G* level of theory.

Zero-point correction $=0.834184($ Hartree/Particle $)$

Thermal correction to Energy $=0.890242$

Thermal correction to Enthalpy $=0.891186$

Thermal correction to Gibbs Free Energy $=0.736072$

Sum of electronic and zero-point Energies $=-2665.405928$

Sum of electronic and thermal Energies $=-2665.349870$

Sum of electronic and thermal Enthalpies $=-2665.348926$

Sum of electronic and thermal Free Energies $=-2665.504041$

NImag $=0$

\begin{tabular}{lrll}
\hline & $x$ & $y$ & $z$ \\
\hline $\mathrm{C}$ & 1.189774 & 0.795155 & 0.081653 \\
$\mathrm{C}$ & 2.358846 & 1.616968 & 0.156750 \\
$\mathrm{H}$ & 3.328225 & 1.130400 & 0.173781 \\
$\mathrm{C}$ & 2.257700 & 2.977482 & 0.201692 \\
$\mathrm{C}$ & 3.259692 & 4.030147 & 0.271874 \\
$\mathrm{C}$ & 4.648607 & 3.997912 & 0.334868 \\
$\mathrm{H}$ & 5.178825 & 3.048983 & 0.329093 \\
$\mathrm{C}$ & 5.361130 & 5.196576 & 0.409453 \\
$\mathrm{H}$ & 6.445935 & 5.181016 & 0.457875 \\
$\mathrm{C}$ & 4.678514 & 6.422094 & 0.427443 \\
$\mathrm{H}$ & 5.245902 & 7.347060 & 0.493150 \\
$\mathrm{C}$ & 3.289078 & 6.475534 & 0.367726 \\
$\mathrm{H}$ & 2.775738 & 7.432528 & 0.397333 \\
$\mathrm{C}$ & 2.556195 & 5.279357 & 0.281221 \\
$\mathrm{C}$ & 1.137296 & 5.030289 & 0.223218 \\
$\mathrm{C}$ & 0.953973 & 3.631405 & 0.175817 \\
$\mathrm{C}$ & -0.196945 & 2.839657 & 0.088523 \\
$\mathrm{H}$ & -1.173867 & 3.307240 & 0.043475 \\
$\mathrm{C}$ & -0.111454 & 1.440136 & 0.044501 \\
$\mathrm{C}$ & 0.072835 & 6.060410 & 0.217845 \\
$\mathrm{C}$ & -0.757389 & 6.235882 & 1.349141 \\
$\mathrm{C}$ & -1.760125 & 7.209662 & 1.322031 \\
$\mathrm{H}$ & -2.391593 & 7.344487 & 2.196500 \\
$\mathrm{C}$ & -1.950501 & 8.012269 & 0.202516 \\
$\mathrm{C}$ & -1.133071 & 7.842856 & -0.909501 \\
$\mathrm{H}$ & -1.285526 & 8.460824 & -1.790842 \\
$\mathrm{C}$ & -0.124187 & 6.875917 & -0.920686 \\
$\mathrm{H}$ & 0.490183 & 5.376935 & 2.891588 \\
$\mathrm{H}$ & -2.733516 & 8.765537 & 0.196377 \\
$\mathrm{Si}$ & -5.249753 & 2.658241 & -0.171683 \\
$\mathrm{C}$ & -1.281339 & 0.610233 & -0.038926 \\
$\mathrm{C}$ & -2.556455 & 1.234245 & -0.077451 \\
\hline & & &
\end{tabular}




\begin{tabular}{|c|c|c|c|}
\hline $\mathrm{C}$ & -3.640462 & 1.790724 & -0.111100 \\
\hline $\mathrm{C}$ & -6.642501 & 1.383047 & -0.100016 \\
\hline $\mathrm{H}$ & -6.587167 & 0.685596 & -0.940821 \\
\hline $\mathrm{H}$ & -6.594508 & 0.791853 & 0.818964 \\
\hline $\mathrm{H}$ & -7.625164 & 1.865725 & -0.133574 \\
\hline $\mathrm{C}$ & -5.386358 & 3.834013 & 1.299659 \\
\hline $\mathrm{H}$ & -4.589616 & 4.583004 & 1.282597 \\
\hline $\mathrm{H}$ & -5.309718 & 3.294854 & 2.248157 \\
\hline $\mathrm{H}$ & -6.343853 & 4.366264 & 1.294523 \\
\hline $\mathrm{C}$ & -5.373997 & 3.642771 & -1.778682 \\
\hline $\mathrm{H}$ & -6.330530 & 4.172352 & -1.847268 \\
\hline $\mathrm{H}$ & -5.290594 & 2.990130 & -2.652342 \\
\hline $\mathrm{H}$ & -4.576037 & 4.387227 & -1.848608 \\
\hline $\mathrm{C}$ & -0.560727 & 5.401320 & 2.592565 \\
\hline $\mathrm{H}$ & -0.861166 & 4.361428 & 2.436265 \\
\hline $\mathrm{H}$ & -1.147590 & 5.797539 & 3.425407 \\
\hline $\mathrm{C}$ & 0.718940 & 6.688920 & -2.159966 \\
\hline $\mathrm{H}$ & 0.329814 & 7.283356 & -2.990811 \\
\hline $\mathrm{H}$ & 0.739741 & 5.640363 & -2.467817 \\
\hline $\mathrm{H}$ & 1.759888 & 6.982144 & -1.995378 \\
\hline $\mathrm{C}$ & -1.189774 & -0.795155 & -0.081653 \\
\hline $\mathrm{C}$ & -2.358846 & -1.616968 & -0.156750 \\
\hline $\mathrm{H}$ & -3.328225 & -1.130400 & -0.173781 \\
\hline $\mathrm{C}$ & -2.257700 & -2.977482 & -0.201692 \\
\hline $\mathrm{C}$ & -3.259692 & -4.030147 & -0.271874 \\
\hline $\mathrm{C}$ & -4.648607 & -3.997912 & -0.334868 \\
\hline $\mathrm{H}$ & -5.178825 & -3.048983 & -0.329093 \\
\hline $\mathrm{C}$ & -5.361130 & -5.196576 & -0.409453 \\
\hline $\mathrm{H}$ & -6.445935 & -5.181016 & -0.457875 \\
\hline $\mathrm{C}$ & -4.678514 & -6.422094 & -0.427443 \\
\hline $\mathrm{H}$ & -5.245902 & -7.347060 & -0.493150 \\
\hline $\mathrm{C}$ & -3.289078 & -6.475534 & -0.367726 \\
\hline $\mathrm{H}$ & -2.775738 & -7.432528 & -0.397333 \\
\hline $\mathrm{C}$ & -2.556195 & -5.279357 & -0.281221 \\
\hline $\mathrm{C}$ & -1.137296 & -5.030289 & -0.223218 \\
\hline $\mathrm{C}$ & -0.953973 & -3.631405 & -0.175817 \\
\hline $\mathrm{C}$ & 0.196945 & -2.839657 & -0.088523 \\
\hline $\mathrm{H}$ & 1.173867 & -3.307240 & -0.043475 \\
\hline $\mathrm{C}$ & 0.111454 & -1.440136 & -0.044501 \\
\hline $\mathrm{C}$ & -0.072835 & -6.060410 & -0.217845 \\
\hline $\mathrm{C}$ & 0.757389 & -6.235882 & -1.349141 \\
\hline $\mathrm{C}$ & 1.760125 & -7.209662 & -1.322031 \\
\hline $\mathrm{H}$ & 2.391593 & -7.344487 & -2.196500 \\
\hline $\mathrm{C}$ & 1.950501 & -8.012269 & -0.202516 \\
\hline $\mathrm{C}$ & 1.133071 & -7.842856 & 0.909501 \\
\hline
\end{tabular}




\begin{tabular}{rrrr}
\hline $\mathrm{H}$ & 1.285526 & -8.460824 & 1.790842 \\
$\mathrm{C}$ & 0.124187 & -6.875917 & 0.920686 \\
$\mathrm{H}$ & -0.490183 & -5.376935 & -2.891588 \\
$\mathrm{H}$ & 2.733516 & -8.765537 & -0.196377 \\
$\mathrm{Si}$ & 5.249753 & -2.658241 & 0.171683 \\
$\mathrm{C}$ & 1.281339 & -0.610233 & 0.038926 \\
$\mathrm{C}$ & 2.556455 & -1.234245 & 0.077451 \\
$\mathrm{C}$ & 3.640462 & -1.790724 & 0.111100 \\
$\mathrm{C}$ & 6.642501 & -1.383047 & 0.100016 \\
$\mathrm{H}$ & 6.587167 & -0.685596 & 0.940821 \\
$\mathrm{H}$ & 6.594508 & -0.791853 & -0.818964 \\
$\mathrm{H}$ & 7.625164 & -1.865725 & 0.133574 \\
$\mathrm{C}$ & 5.386358 & -3.834013 & -1.299659 \\
$\mathrm{H}$ & 4.589616 & -4.583004 & -1.282597 \\
$\mathrm{H}$ & 5.309718 & -3.294854 & -2.248157 \\
$\mathrm{H}$ & 6.343853 & -4.366264 & -1.294523 \\
$\mathrm{C}$ & 5.373997 & -3.642771 & 1.778682 \\
$\mathrm{H}$ & 6.330530 & -4.172352 & 1.847268 \\
$\mathrm{H}$ & 5.290594 & -2.990130 & 2.652342 \\
$\mathrm{H}$ & 4.576037 & -4.387227 & 1.848608 \\
$\mathrm{C}$ & 0.560727 & -5.401320 & -2.592565 \\
$\mathrm{H}$ & 0.861166 & -4.361428 & -2.436265 \\
$\mathrm{H}$ & 1.147590 & -5.797539 & -3.425407 \\
$\mathrm{C}$ & -0.718940 & -6.688920 & 2.159966 \\
$\mathrm{H}$ & -0.329814 & -7.283356 & 2.990811 \\
$\mathrm{H}$ & -0.739741 & -5.640363 & 2.467817 \\
$\mathrm{H}$ & -1.759888 & -6.982144 & 1.995378 \\
\hline & & & \\
\hline
\end{tabular}


Table S6. Optimized geometry of DIAn ${ }^{2+}$ calculated at RB3LYP/6-311G* level of theory.

Zero-point correction $=0.839521$ (Hartree/Particle $)$

Thermal correction to Energy $=0.895793$

Thermal correction to Enthalpy $=0.896737$

Thermal correction to Gibbs Free Energy $=0.741682$

Sum of electronic and zero-point Energies $=-2664.797085$

Sum of electronic and thermal Energies $=-2664.740813$

Sum of electronic and thermal Enthalpies $=-2664.739869$

Sum of electronic and thermal Free Energies $=-2664.894924$

NImag $=0$

\begin{tabular}{lrlc}
\hline & $x$ & $y$ & $z$ \\
\hline $\mathrm{C}$ & 1.199236 & 0.775802 & 0.070328 \\
$\mathrm{C}$ & 2.388828 & 1.580722 & 0.120795 \\
$\mathrm{H}$ & 3.348576 & 1.078656 & 0.100223 \\
$\mathrm{C}$ & 2.292344 & 2.936138 & 0.174602 \\
$\mathrm{C}$ & 3.294257 & 4.006721 & 0.185213 \\
$\mathrm{C}$ & 4.675841 & 3.987810 & 0.229721 \\
$\mathrm{H}$ & 5.231922 & 3.057321 & 0.246073 \\
$\mathrm{C}$ & 5.358593 & 5.212461 & 0.268519 \\
$\mathrm{H}$ & 6.442573 & 5.211134 & 0.305290 \\
$\mathrm{C}$ & 4.678359 & 6.440333 & 0.281553 \\
$\mathrm{H}$ & 5.243875 & 7.363034 & 0.333724 \\
$\mathrm{C}$ & 3.293240 & 6.474273 & 0.242036 \\
$\mathrm{H}$ & 2.759659 & 7.416678 & 0.277567 \\
$\mathrm{C}$ & 2.592223 & 5.255039 & 0.172316 \\
$\mathrm{C}$ & 1.184612 & 5.012842 & 0.187662 \\
$\mathrm{C}$ & 1.001809 & 3.574445 & 0.188244 \\
$\mathrm{C}$ & -0.153712 & 2.834893 & 0.096909 \\
$\mathrm{H}$ & -1.123095 & 3.316748 & 0.057604 \\
$\mathrm{C}$ & -0.086558 & 1.413138 & 0.044620 \\
$\mathrm{C}$ & 0.128657 & 6.017176 & 0.200929 \\
$\mathrm{C}$ & -0.775116 & 6.068104 & 1.301503 \\
$\mathrm{C}$ & -1.752265 & 7.059428 & 1.305288 \\
$\mathrm{H}$ & -2.415095 & 7.143635 & 2.160228 \\
$\mathrm{C}$ & -1.875592 & 7.957267 & 0.248076 \\
$\mathrm{C}$ & -1.002248 & 7.888684 & -0.831300 \\
$\mathrm{H}$ & -1.117474 & 8.578877 & -1.660593 \\
$\mathrm{C}$ & 0.021289 & 6.943983 & -0.874939 \\
$\mathrm{H}$ & 0.357331 & 4.815527 & 2.691745 \\
$\mathrm{H}$ & -2.650789 & 8.715460 & 0.269690 \\
$\mathrm{Si}$ & -5.314997 & 2.650780 & -0.193353 \\
$\mathrm{C}$ & -1.283523 & 0.638321 & -0.029516 \\
$\mathrm{C}$ & -2.545496 & 1.279022 & -0.067130 \\
\hline & & &
\end{tabular}




\begin{tabular}{|c|c|c|c|}
\hline $\mathrm{C}$ & -3.633590 & 1.825283 & -0.109701 \\
\hline $\mathrm{C}$ & -6.600981 & 1.281265 & -0.147091 \\
\hline $\mathrm{H}$ & -6.499986 & 0.600298 & -0.996827 \\
\hline $\mathrm{H}$ & -6.537055 & 0.692422 & 0.772114 \\
\hline $\mathrm{H}$ & -7.609535 & 1.703197 & -0.190693 \\
\hline $\mathrm{C}$ & -5.448439 & 3.782791 & 1.300801 \\
\hline $\mathrm{H}$ & -4.681817 & 4.562514 & 1.289755 \\
\hline $\mathrm{H}$ & -5.355599 & 3.233281 & 2.241164 \\
\hline $\mathrm{H}$ & -6.421211 & 4.283809 & 1.311454 \\
\hline $\mathrm{C}$ & -5.374414 & 3.611734 & -1.806447 \\
\hline $\mathrm{H}$ & -6.342731 & 4.109787 & -1.915404 \\
\hline $\mathrm{H}$ & -5.243377 & 2.961460 & -2.675174 \\
\hline $\mathrm{H}$ & -4.603494 & 4.385694 & -1.850086 \\
\hline $\mathrm{C}$ & -0.659613 & 5.166161 & 2.512694 \\
\hline $\mathrm{H}$ & -1.301816 & 4.284624 & 2.427539 \\
\hline $\mathrm{H}$ & -0.978314 & 5.700870 & 3.408650 \\
\hline $\mathrm{C}$ & 0.902378 & 6.903493 & -2.105033 \\
\hline $\mathrm{H}$ & 0.308519 & 7.108900 & -2.997504 \\
\hline $\mathrm{H}$ & 1.394795 & 5.943406 & -2.256629 \\
\hline $\mathrm{H}$ & 1.680950 & 7.670972 & -2.065639 \\
\hline $\mathrm{C}$ & -1.199236 & -0.775802 & -0.070328 \\
\hline $\mathrm{C}$ & -2.388828 & -1.580722 & -0.120795 \\
\hline $\mathrm{H}$ & -3.348576 & -1.078656 & -0.100223 \\
\hline $\mathrm{C}$ & -2.292344 & -2.936138 & -0.174602 \\
\hline $\mathrm{C}$ & -3.294257 & -4.006721 & -0.185213 \\
\hline $\mathrm{C}$ & -4.675841 & -3.987810 & -0.229721 \\
\hline $\mathrm{H}$ & -5.231922 & -3.057321 & -0.246073 \\
\hline $\mathrm{C}$ & -5.358593 & -5.212461 & -0.268519 \\
\hline $\mathrm{H}$ & -6.442573 & -5.211134 & -0.305290 \\
\hline $\mathrm{C}$ & -4.678359 & -6.440333 & -0.281553 \\
\hline $\mathrm{H}$ & -5.243875 & -7.363034 & -0.333724 \\
\hline $\mathrm{C}$ & -3.293240 & -6.474273 & -0.242036 \\
\hline $\mathrm{H}$ & -2.759659 & -7.416678 & -0.277567 \\
\hline $\mathrm{C}$ & -2.592223 & -5.255039 & -0.172316 \\
\hline $\mathrm{C}$ & -1.184612 & -5.012842 & -0.187662 \\
\hline $\mathrm{C}$ & -1.001809 & -3.574445 & -0.188244 \\
\hline $\mathrm{C}$ & 0.153712 & -2.834893 & -0.096909 \\
\hline $\mathrm{H}$ & 1.123095 & -3.316748 & -0.057604 \\
\hline $\mathrm{C}$ & 0.086558 & -1.413138 & -0.044620 \\
\hline $\mathrm{C}$ & -0.128657 & -6.017176 & -0.200929 \\
\hline $\mathrm{C}$ & 0.775116 & -6.068104 & -1.301503 \\
\hline $\mathrm{C}$ & 1.752265 & -7.059428 & -1.305288 \\
\hline $\mathrm{H}$ & 2.415095 & -7.143635 & -2.160228 \\
\hline $\mathrm{C}$ & 1.875592 & -7.957267 & -0.248076 \\
\hline $\mathrm{C}$ & 1.002248 & -7.888684 & 0.831300 \\
\hline
\end{tabular}




\begin{tabular}{rrrr}
\hline $\mathrm{H}$ & 1.117474 & -8.578877 & 1.660593 \\
$\mathrm{C}$ & -0.021289 & -6.943983 & 0.874939 \\
$\mathrm{H}$ & -0.357331 & -4.815527 & -2.691745 \\
$\mathrm{H}$ & 2.650789 & -8.715460 & -0.269690 \\
$\mathrm{Si}$ & 5.314997 & -2.650780 & 0.193353 \\
$\mathrm{C}$ & 1.283523 & -0.638321 & 0.029516 \\
$\mathrm{C}$ & 2.545496 & -1.279022 & 0.067130 \\
$\mathrm{C}$ & 3.633590 & -1.825283 & 0.109701 \\
$\mathrm{C}$ & 6.600981 & -1.281265 & 0.147091 \\
$\mathrm{H}$ & 6.499986 & -0.600298 & 0.996827 \\
$\mathrm{H}$ & 6.537055 & -0.692422 & -0.772114 \\
$\mathrm{H}$ & 7.609535 & -1.703197 & 0.190693 \\
$\mathrm{C}$ & 5.448439 & -3.782791 & -1.300801 \\
$\mathrm{H}$ & 4.681817 & -4.562514 & -1.289755 \\
$\mathrm{H}$ & 5.355599 & -3.233281 & -2.241164 \\
$\mathrm{H}$ & 6.421211 & -4.283809 & -1.311454 \\
$\mathrm{C}$ & 5.374414 & -3.611734 & 1.806447 \\
$\mathrm{H}$ & 6.342731 & -4.109787 & 1.915404 \\
$\mathrm{H}$ & 5.243377 & -2.961460 & 2.675174 \\
$\mathrm{H}$ & 4.603494 & -4.385694 & 1.850086 \\
$\mathrm{C}$ & 0.659613 & -5.166161 & -2.512694 \\
$\mathrm{H}$ & 1.301816 & -4.284624 & -2.427539 \\
$\mathrm{H}$ & 0.978314 & -5.700870 & -3.408650 \\
$\mathrm{C}$ & -0.902378 & -6.903493 & 2.105033 \\
$\mathrm{H}$ & -0.308519 & -7.108900 & 2.997504 \\
$\mathrm{H}$ & -1.394795 & -5.943406 & 2.256629 \\
$\mathrm{H}$ & -1.680950 & -7.670972 & 2.065639 \\
\hline & & &
\end{tabular}


Table S7. Optimized geometry of DIAn ${ }^{2-}$ calculated at RB3LYP/6-311G* level of theory.

Zero-point correction $=0.831428$ (Hartree/Particle $)$

Thermal correction to Energy $=0.887184$

Thermal correction to Enthalpy $=0.888128$

Thermal correction to Gibbs Free Energy $=0.736709$

Sum of electronic and zero-point Energies $=-2665.395095$

Sum of electronic and thermal Energies $=-2665.339339$

Sum of electronic and thermal Enthalpies $=-2665.338395$

Sum of electronic and thermal Free Energies $=-2665.489814$

NImag $=0$

\begin{tabular}{lrlc}
\hline & $x$ & $y$ & $z$ \\
\hline $\mathrm{C}$ & 1.186081 & 0.805294 & 0.078916 \\
$\mathrm{C}$ & 2.345067 & 1.627846 & 0.143700 \\
$\mathrm{H}$ & 3.318942 & 1.148662 & 0.145583 \\
$\mathrm{C}$ & 2.243090 & 2.996093 & 0.191839 \\
$\mathrm{C}$ & 3.249456 & 4.039062 & 0.242614 \\
$\mathrm{C}$ & 4.638509 & 3.991564 & 0.300277 \\
$\mathrm{H}$ & 5.150989 & 3.031342 & 0.297661 \\
$\mathrm{C}$ & 5.376974 & 5.175950 & 0.368486 \\
$\mathrm{H}$ & 6.462820 & 5.144483 & 0.411099 \\
$\mathrm{C}$ & 4.703734 & 6.409508 & 0.392318 \\
$\mathrm{H}$ & 5.281898 & 7.330127 & 0.461517 \\
$\mathrm{C}$ & 3.316185 & 6.484315 & 0.339148 \\
$\mathrm{H}$ & 2.823488 & 7.452120 & 0.391739 \\
$\mathrm{C}$ & 2.548664 & 5.300274 & 0.247781 \\
$\mathrm{C}$ & 1.136804 & 5.069454 & 0.212912 \\
$\mathrm{C}$ & 0.940380 & 3.654574 & 0.180476 \\
$\mathrm{C}$ & -0.201114 & 2.858150 & 0.092270 \\
$\mathrm{H}$ & -1.181315 & 3.317727 & 0.031704 \\
$\mathrm{C}$ & -0.115152 & 1.449239 & 0.047220 \\
$\mathrm{C}$ & 0.088907 & 6.104582 & 0.215610 \\
$\mathrm{C}$ & -0.847119 & 6.194282 & 1.281518 \\
$\mathrm{C}$ & -1.835304 & 7.182233 & 1.263279 \\
$\mathrm{H}$ & -2.537992 & 7.240867 & 2.092159 \\
$\mathrm{C}$ & -1.918870 & 8.102865 & 0.222697 \\
$\mathrm{C}$ & -1.003492 & 8.026966 & -0.822241 \\
$\mathrm{H}$ & -1.073313 & 8.728390 & -1.651855 \\
$\mathrm{C}$ & -0.012046 & 7.042591 & -0.847357 \\
$\mathrm{H}$ & 0.278471 & 5.186032 & 2.815511 \\
$\mathrm{H}$ & -2.692244 & 8.867485 & 0.224296 \\
$\mathrm{Si}$ & -5.247603 & 2.620615 & -0.153143 \\
$\mathrm{C}$ & -1.276246 & 0.612924 & -0.034134 \\
$\mathrm{C}$ & -2.552282 & 1.225602 & -0.066973 \\
\hline & & &
\end{tabular}




\begin{tabular}{|c|c|c|c|}
\hline $\mathrm{C}$ & -3.645902 & 1.770173 & -0.094709 \\
\hline $\mathrm{C}$ & -6.647195 & 1.376348 & 0.131622 \\
\hline $\mathrm{H}$ & -6.638727 & 0.589535 & -0.628324 \\
\hline $\mathrm{H}$ & -6.549002 & 0.886990 & 1.104981 \\
\hline $\mathrm{H}$ & -7.628933 & 1.862740 & 0.098572 \\
\hline $\mathrm{C}$ & -5.332965 & 3.956351 & 1.184246 \\
\hline $\mathrm{H}$ & -4.534825 & 4.693177 & 1.056943 \\
\hline $\mathrm{H}$ & -5.218918 & 3.522268 & 2.181917 \\
\hline $\mathrm{H}$ & -6.290054 & 4.490363 & 1.157398 \\
\hline $\mathrm{C}$ & -5.501748 & 3.440005 & -1.841581 \\
\hline $\mathrm{H}$ & -6.465702 & 3.959376 & -1.896254 \\
\hline $\mathrm{H}$ & -5.472371 & 2.700401 & -2.647133 \\
\hline $\mathrm{H}$ & -4.715015 & 4.172663 & -2.042526 \\
\hline $\mathrm{C}$ & -0.754763 & 5.265287 & 2.466901 \\
\hline $\mathrm{H}$ & -1.067178 & 4.246619 & 2.219857 \\
\hline $\mathrm{H}$ & -1.380640 & 5.620093 & 3.291812 \\
\hline $\mathrm{C}$ & 0.903571 & 6.949609 & -2.044207 \\
\hline $\mathrm{H}$ & 0.533841 & 7.569068 & -2.867834 \\
\hline $\mathrm{H}$ & 0.981521 & 5.915354 & -2.389144 \\
\hline $\mathrm{H}$ & 1.925634 & 7.267326 & -1.815119 \\
\hline $\mathrm{C}$ & -1.186081 & -0.805294 & -0.078916 \\
\hline $\mathrm{C}$ & -2.345067 & -1.627846 & -0.143700 \\
\hline $\mathrm{H}$ & -3.318942 & -1.148662 & -0.145583 \\
\hline $\mathrm{C}$ & -2.243090 & -2.996093 & -0.191839 \\
\hline $\mathrm{C}$ & -3.249456 & -4.039062 & -0.242614 \\
\hline $\mathrm{C}$ & -4.638509 & -3.991564 & -0.300277 \\
\hline $\mathrm{H}$ & -5.150989 & -3.031342 & -0.297661 \\
\hline $\mathrm{C}$ & -5.376974 & -5.175950 & -0.368486 \\
\hline $\mathrm{H}$ & -6.462820 & -5.144483 & -0.411099 \\
\hline $\mathrm{C}$ & -4.703734 & -6.409508 & -0.392318 \\
\hline $\mathrm{H}$ & -5.281898 & -7.330127 & -0.461517 \\
\hline $\mathrm{C}$ & -3.316185 & -6.484315 & -0.339148 \\
\hline $\mathrm{H}$ & -2.823488 & -7.452120 & -0.391739 \\
\hline $\mathrm{C}$ & -2.548664 & -5.300274 & -0.247781 \\
\hline $\mathrm{C}$ & -1.136804 & -5.069454 & -0.212912 \\
\hline $\mathrm{C}$ & -0.940380 & -3.654574 & -0.180476 \\
\hline $\mathrm{C}$ & 0.201114 & -2.858150 & -0.092270 \\
\hline $\mathrm{H}$ & 1.181315 & -3.317727 & -0.031704 \\
\hline $\mathrm{C}$ & 0.115152 & -1.449239 & -0.047220 \\
\hline $\mathrm{C}$ & -0.088907 & -6.104582 & -0.215610 \\
\hline $\mathrm{C}$ & 0.847119 & -6.194282 & -1.281518 \\
\hline $\mathrm{C}$ & 1.835304 & -7.182233 & -1.263279 \\
\hline $\mathrm{H}$ & 2.537992 & -7.240867 & -2.092159 \\
\hline $\mathrm{C}$ & 1.918870 & -8.102865 & -0.222697 \\
\hline $\mathrm{C}$ & 1.003492 & -8.026966 & 0.822241 \\
\hline
\end{tabular}




\begin{tabular}{rrrr}
\hline $\mathrm{H}$ & 1.073313 & -8.728390 & 1.651855 \\
$\mathrm{C}$ & 0.012046 & -7.042591 & 0.847357 \\
$\mathrm{H}$ & -0.278471 & -5.186032 & -2.815511 \\
$\mathrm{H}$ & 2.692244 & -8.867485 & -0.224296 \\
$\mathrm{Si}$ & 5.247603 & -2.620615 & 0.153143 \\
$\mathrm{C}$ & 1.276246 & -0.612924 & 0.034134 \\
$\mathrm{C}$ & 2.552282 & -1.225602 & 0.066973 \\
$\mathrm{C}$ & 3.645902 & -1.770173 & 0.094709 \\
$\mathrm{C}$ & 6.647195 & -1.376348 & -0.131622 \\
$\mathrm{H}$ & 6.638727 & -0.589535 & 0.628324 \\
$\mathrm{H}$ & 6.549002 & -0.886990 & -1.104981 \\
$\mathrm{H}$ & 7.628933 & -1.862740 & -0.098572 \\
$\mathrm{C}$ & 5.332965 & -3.956351 & -1.184246 \\
$\mathrm{H}$ & 4.534825 & -4.693177 & -1.056943 \\
$\mathrm{H}$ & 5.218918 & -3.522268 & -2.181917 \\
$\mathrm{H}$ & 6.290054 & -4.490363 & -1.157398 \\
$\mathrm{C}$ & 5.501748 & -3.440005 & 1.841581 \\
$\mathrm{H}$ & 6.465702 & -3.959376 & 1.896254 \\
$\mathrm{H}$ & 5.472371 & -2.700401 & 2.647133 \\
$\mathrm{H}$ & 4.715015 & -4.172663 & 2.042526 \\
$\mathrm{C}$ & 0.754763 & -5.265287 & -2.466901 \\
$\mathrm{H}$ & 1.067178 & -4.246619 & -2.219857 \\
$\mathrm{H}$ & 1.380640 & -5.620093 & -3.291812 \\
$\mathrm{C}$ & -0.903571 & -6.949609 & 2.044207 \\
$\mathrm{H}$ & -0.533841 & -7.569068 & 2.867834 \\
$\mathrm{H}$ & -0.981521 & -5.915354 & 2.389144 \\
$\mathrm{H}$ & -1.925634 & -7.267326 & 1.815119 \\
\hline & & & \\
\hline
\end{tabular}




\section{TD-DFT Calculations}

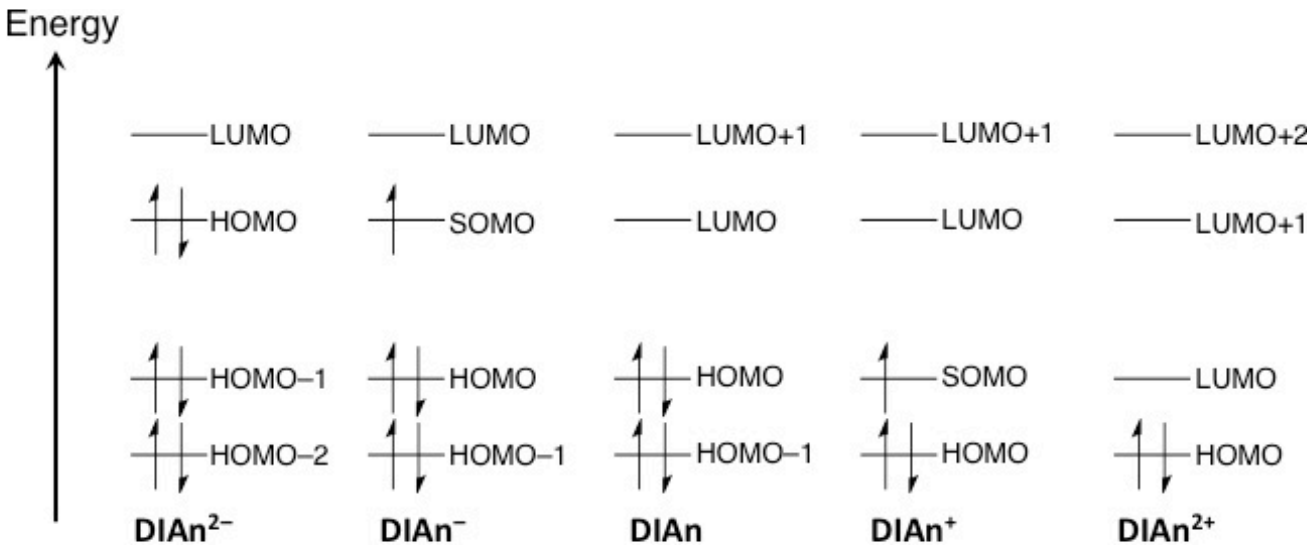

Figure S21. Orbital diagrams of DIAn series.

Table S8. TD-UB3LYP/6-311+G** result of low lying states with non-zero $\mathrm{f}$ in neutral state.

\begin{tabular}{|c|c|c|c|}
\hline state & Configuration & $\mathrm{E}$ & $\mathrm{f}$ \\
\hline 2 & $\begin{array}{l}\mathrm{H}-1 \mathrm{a}=>\mathrm{La}(0.43) \\
\mathrm{Ha}=>\mathrm{La}(-0.28) \\
\mathrm{Ha}=>\mathrm{L}+1 \mathrm{a}(0.46) \\
\mathrm{H}-1 \mathrm{a}=>\mathrm{Lb}(-0.43) \\
\mathrm{Hb}=>\mathrm{Lb}(0.28) \\
\mathrm{Hb}=>\mathrm{L}+1 \mathrm{~b}(0.46)\end{array}$ & $\begin{array}{c}1.5490 \mathrm{eV} \\
(800.43 \mathrm{~nm})\end{array}$ & 0.0950 \\
\hline 3 & $\begin{array}{l}\mathrm{H}-1 \mathrm{a}=>\mathrm{La}(-0.14) \\
\mathrm{H}-1 \mathrm{a}=>\mathrm{L}+1 \mathrm{a}(0.13) \\
\mathrm{Ha}=>\mathrm{La}(-0.62) \\
\mathrm{Ha}=>\mathrm{L}+1 \mathrm{a}(-0.27) \\
\mathrm{H}-1 \mathrm{~b}=>\mathrm{Lb}(0.14) \\
\mathrm{H}-1 \mathrm{~b}=>\mathrm{L}+1 \mathrm{~b}(0.13) \\
\mathrm{Hb}=>\mathrm{Lb}(0.62) \\
\mathrm{Hb}=>\mathrm{L}+1 \mathrm{~b}(-0.27)\end{array}$ & $\begin{array}{c}1.7264 \mathrm{eV} \\
(718.17 \mathrm{~nm})\end{array}$ & 0.5228 \\
\hline
\end{tabular}

Table S9. TD-UB3LYP/6-311+G** result of low lying states with non-zero $\mathrm{f}$ in mono-anionic state.

\begin{tabular}{clcc}
\hline state & \multicolumn{1}{c}{ configuration } & $\mathrm{E}$ & $\mathrm{f}$ \\
\hline \multirow{4}{*}{1} & $\mathrm{H}-1 \mathrm{a}=>\mathrm{La}(-0.10)$ & $0.8595 \mathrm{eV}$ & \\
& $\mathrm{Sa}=>\mathrm{La}(0.51)$ & $(1442.58 \mathrm{~nm})$ & 0.1841 \\
& $\mathrm{Hb}=>\mathrm{Sb}(0.84)$ & & \\
& $\mathrm{H}-1 \mathrm{a}=>\mathrm{La}(0.17)$ & $1.0229 \mathrm{eV}$ & \\
\multirow{2}{*}{2} & $\mathrm{Sa}=>\mathrm{La}(0.84)$ & $(1212.05 \mathrm{~nm})$ & 0.2244 \\
& $\mathrm{H}-2 \mathrm{~b}=>\mathrm{Lb}(0.11)$ & & \\
\hline
\end{tabular}


Table S10. TD-U(R)B3LYP/6-311+G** result of low lying states with non-zero $f$ in dianionic state [restricted wavefunction]

\begin{tabular}{clcc}
\hline state & configuration & $\mathrm{E}$ & $\mathrm{f}$ \\
\hline \multirow{2}{*}{2} & $\mathrm{Ha}=>\mathrm{La}(0.71)$ & $0.9589 \mathrm{eV}$ & \\
& $\mathrm{Hb}=>\mathrm{Lb}(-0.71)$ & $(1293.00 \mathrm{~nm})$ & 0.0785 \\
\hline
\end{tabular}

Table S11. TD-UB3LYP/6-311+G** result of low lying states with non-zero $f$ in monocationic state

\begin{tabular}{clcc}
\hline state & \multicolumn{1}{c}{ configuration } & $\mathrm{E}$ & $\mathrm{f}$ \\
\hline \multirow{3}{*}{1} & $\mathrm{Sa}=>\mathrm{La}(0.90)$ & & \\
$\mathrm{H}-1 \mathrm{~b}=>\mathrm{Sb}(-0.13)$ & $(1454.59 \mathrm{~nm})$ & 0.1663 \\
& $\mathrm{Hb}=>\mathrm{Sb}(0.39)$ & & \\
& $\mathrm{Hb}=>\mathrm{L}+1 \mathrm{~b}(-0.12)$ & & \\
& $\mathrm{Sa}=>\mathrm{La}(-0.31)$ & & \\
& $\mathrm{H}-6 \mathrm{~b}=>\mathrm{Lb}(-0.12)$ & & \\
3 & $\mathrm{H}-3 \mathrm{~b}=>\mathrm{Sb}(-0.20)$ & $(940.87 \mathrm{~nm})$ & \\
& $\mathrm{H}-1 \mathrm{~b}=>\mathrm{Sb}(-0.30)$ & & \\
& $\mathrm{Hb}=>\mathrm{Sb}(0.87)$ & & \\
\hline
\end{tabular}

Table S12. TD-U(R)B3LYP/6-311+G** result of low lying states with non-zero $f$ in dicationic state [restricted wavefunction]

\begin{tabular}{clcc}
\hline state & \multicolumn{1}{c}{ configuration } & $\mathrm{E}$ & $\mathrm{f}$ \\
\hline \multirow{2}{*}{2} & $\mathrm{Ha}=>\mathrm{La}(0.68)$ & & \\
$\mathrm{Hb}=>\mathrm{Lb}(0.68)$ & $1.0894 \mathrm{eV}$ & 0.1811 \\
& $\mathrm{H}-3 \mathrm{a}=>\mathrm{La}(0.19)$ & $(1138.13 \mathrm{~nm})$ & \\
\hline $\mathrm{H}-3 \mathrm{~b}=>\mathrm{Lb}(0.19)$ & & \\
\hline
\end{tabular}




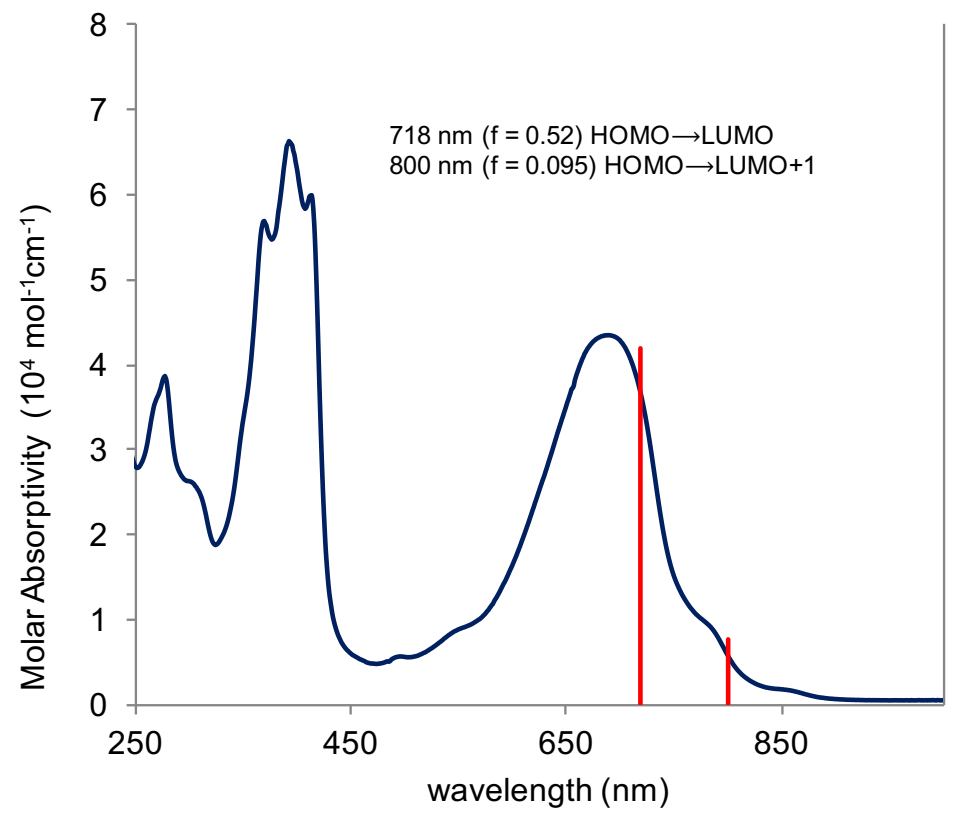

Figure S22. Electronic absorption spectra of neutral DIAn and and electronic transitions from TDDFT calculation.

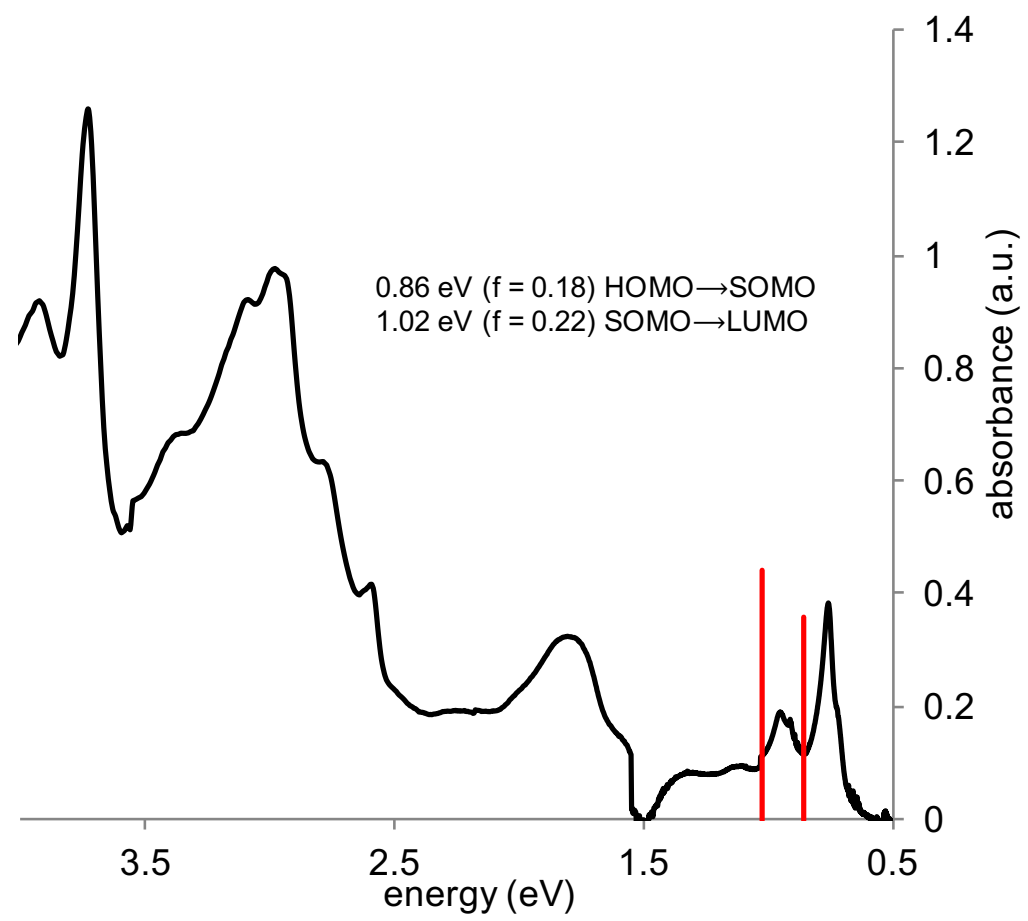

Figure S23. DIAn radical anion generated by electrolysis and electronic transitions from TDDFT calculation. 


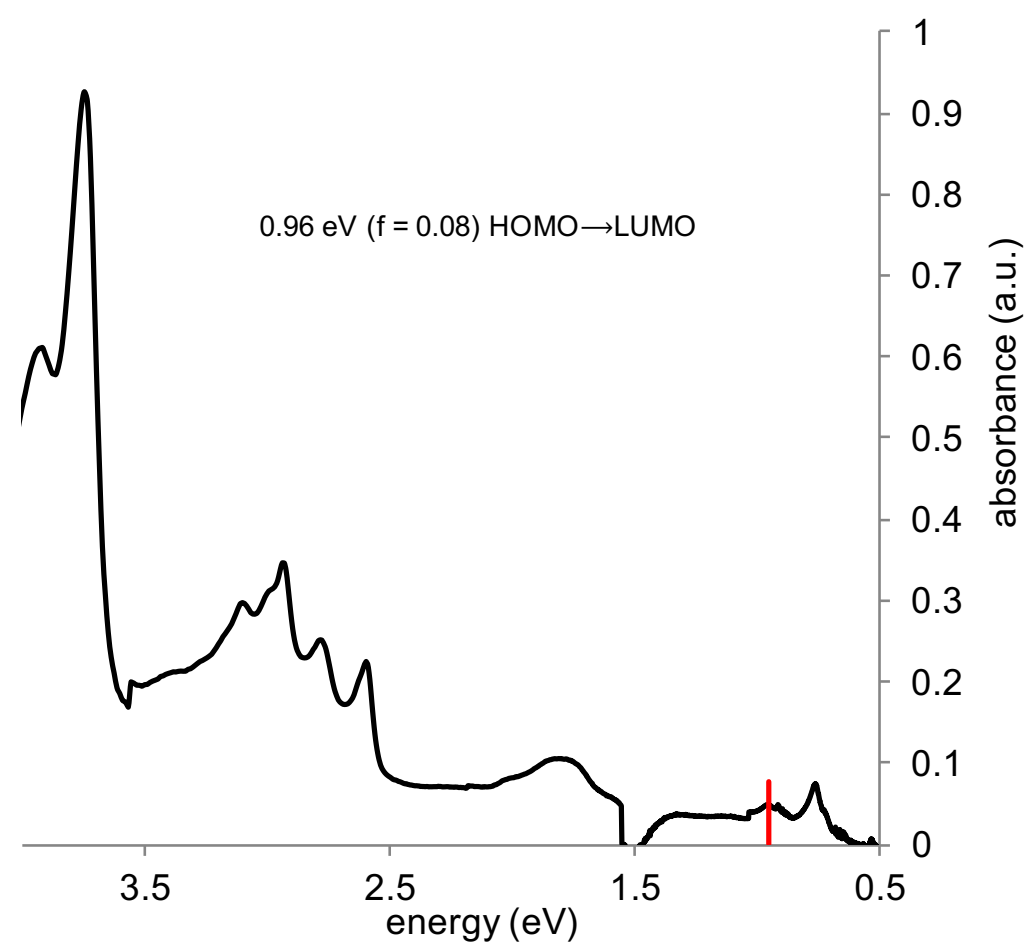

Figure S24. DIAn dianion generated by electrolysis and electronic transitions from TDDFT calculation.

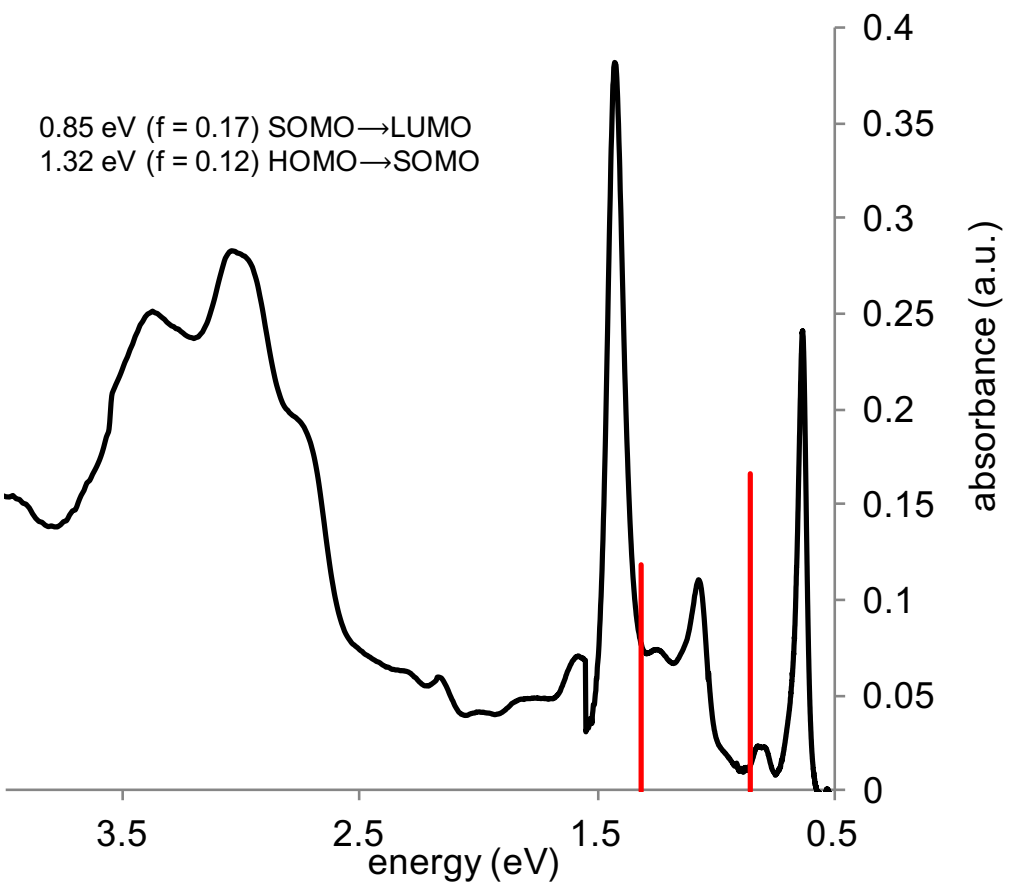

Figure S25. DIAn radical cation generated by electrolysis and electronic transitions from TDDFT calculation. 


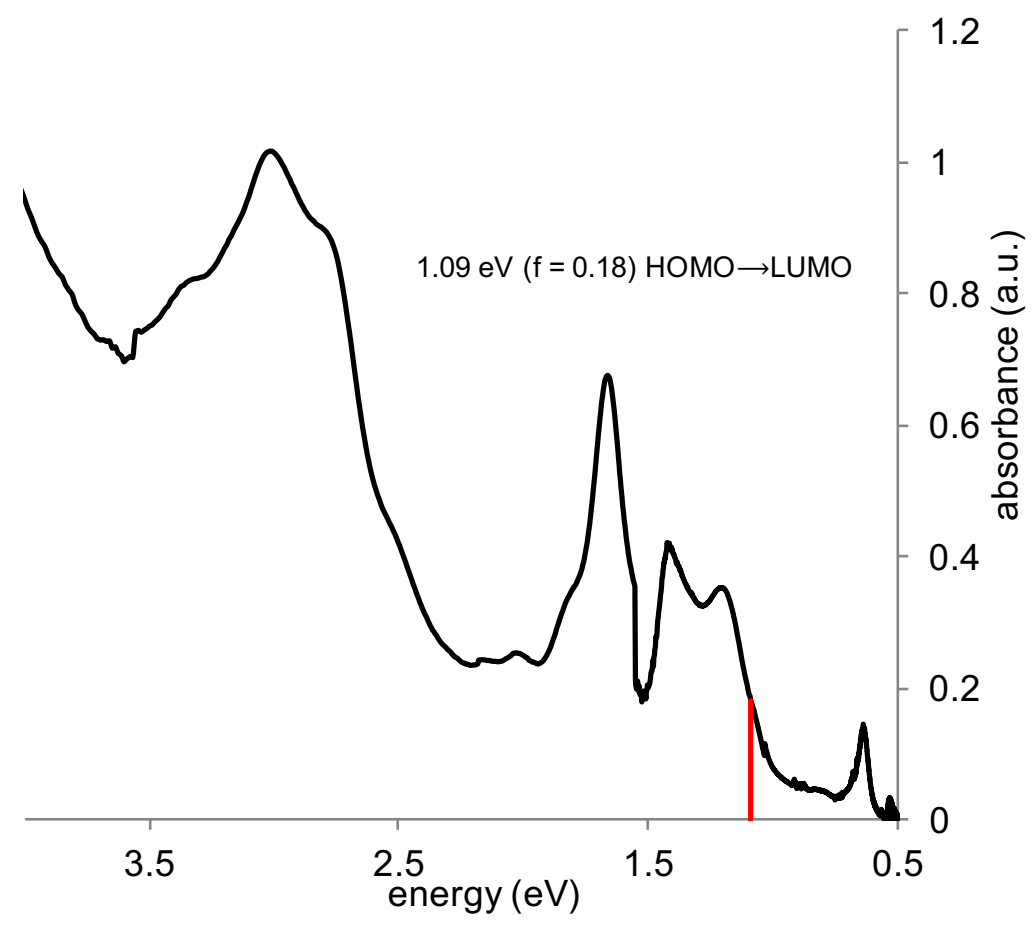

Figure S26. DIAn dication generated by electrolysis and electronic transitions from TDDFT calculation. 


\section{Hirshfeld Charge}

Atomic Hirshfeld charges are also calculated at LC-UBLYP/6-311+G** level of theory for each optimized geometry (Table S8). It is found that the largest difference upon ionization occurs at the carbon atoms of the five-membered rings at which the dimethylphenyl substituents are bonded (atom number 15 and 67 in Figure S26). More exactly, the carbon atom in the cationic DIAn ${ }^{+}$system exhibits 0.11 a.u. less, and that in the anionic DIAn ${ }^{-}$system does 0.11 more electrons. This observation also substantiates the charge localization at the five-membered rings in mono-ionic states.

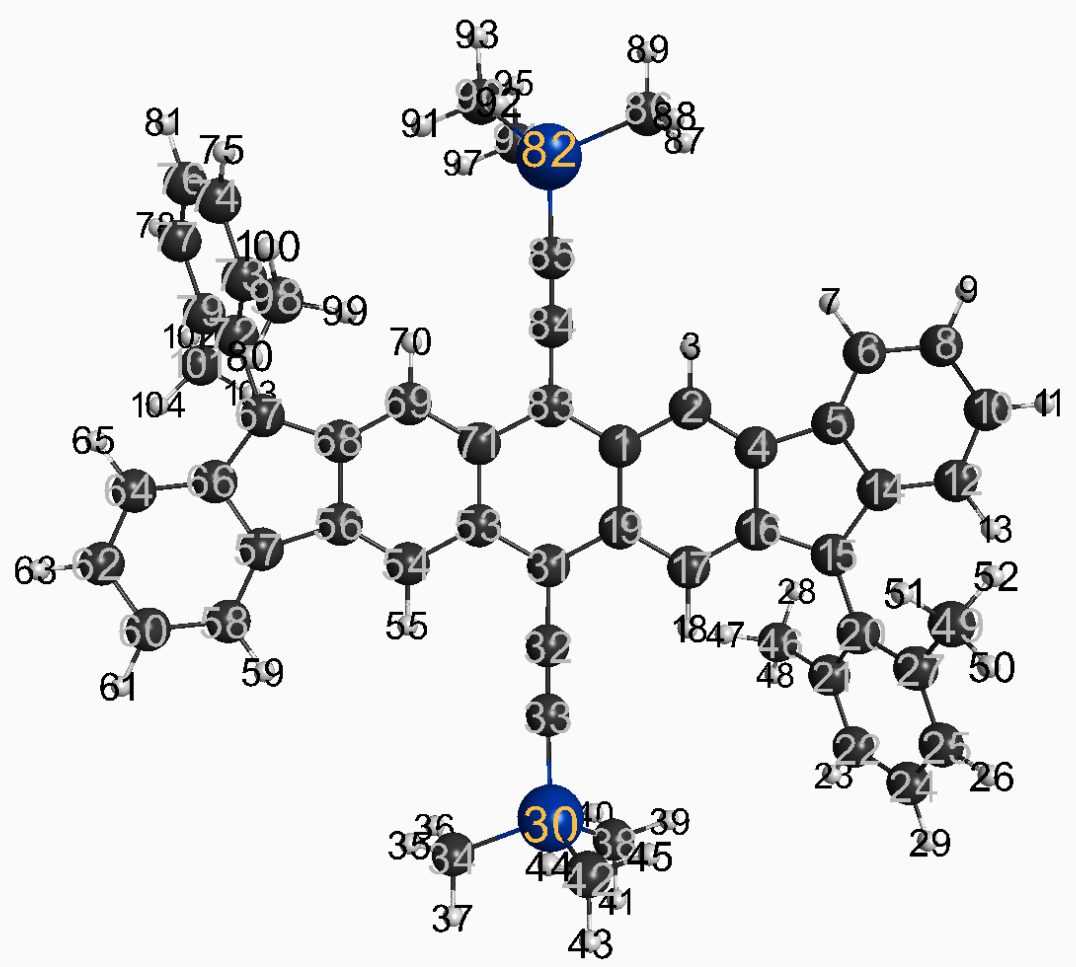

Figure S27. Atomic numbering.

Table S13. Hirshfeld charges of each state calculated at LC-UBLYP/6-311+G** method.

\begin{tabular}{rrrr}
\hline Atom Number & \multicolumn{1}{c}{ DIAn $^{+}$} & \multicolumn{1}{c}{ DIAn } & \multicolumn{1}{c}{ DIAn $^{-}$} \\
\hline 1 & 0.017406 & 0.000852 & -0.020148 \\
2 & -0.004518 & -0.029016 & -0.060802 \\
3 & 0.064263 & 0.049505 & 0.031908 \\
4 & -0.003251 & -0.009163 & -0.028217 \\
5 & 0.028278 & -0.012766 & -0.047212 \\
6 & -0.015471 & -0.039281 & -0.063385 \\
7 & 0.069401 & 0.049597 & 0.031467 \\
8 & 0.032272 & -0.046990 & -0.115948 \\
9 & 0.079963 & 0.045302 & 0.010180 \\
10 & -0.012840 & -0.047294 & -0.084501 \\
11 & 0.074408 & 0.045632 & 0.016346 \\
\hline
\end{tabular}




\begin{tabular}{|c|c|c|c|}
\hline 12 & 0.019351 & -0.040857 & -0.086072 \\
\hline 13 & 0.075162 & 0.050323 & 0.024874 \\
\hline 14 & -0.012184 & -0.015863 & -0.023090 \\
\hline 15 & 0.122832 & 0.010704 & -0.103894 \\
\hline 16 & -0.010212 & -0.021843 & -0.025473 \\
\hline 17 & 0.033220 & -0.028300 & -0.090364 \\
\hline 18 & 0.069335 & 0.049098 & 0.026864 \\
\hline 19 & 0.012613 & -0.006389 & -0.017872 \\
\hline 20 & -0.024164 & -0.017272 & -0.006787 \\
\hline 21 & 0.014528 & 0.000052 & -0.007537 \\
\hline 22 & -0.036601 & -0.057218 & -0.075782 \\
\hline 23 & 0.061114 & 0.042262 & 0.025646 \\
\hline 24 & -0.004804 & -0.047889 & -0.082799 \\
\hline 25 & -0.034391 & -0.057306 & -0.079000 \\
\hline 26 & 0.063366 & 0.042184 & 0.022896 \\
\hline 27 & 0.017251 & -0.000267 & -0.010854 \\
\hline 28 & 0.041852 & 0.042174 & 0.037770 \\
\hline 29 & 0.070106 & 0.045627 & 0.022686 \\
\hline 30 & 0.422340 & 0.414828 & 0.403890 \\
\hline 31 & 0.030542 & 0.007240 & -0.022117 \\
\hline 32 & -0.056913 & -0.036753 & -0.016017 \\
\hline 33 & -0.122158 & -0.160376 & -0.203130 \\
\hline 34 & -0.189076 & -0.194486 & -0.200641 \\
\hline 35 & 0.039738 & 0.037171 & 0.033574 \\
\hline 36 & 0.040416 & 0.037420 & 0.032878 \\
\hline 37 & 0.050563 & 0.036526 & 0.021795 \\
\hline 38 & -0.188632 & -0.193625 & -0.199576 \\
\hline 39 & 0.037559 & 0.039184 & 0.038712 \\
\hline 40 & 0.043559 & 0.038254 & 0.031677 \\
\hline 41 & 0.049675 & 0.036062 & 0.021966 \\
\hline 42 & -0.187586 & -0.193405 & -0.199996 \\
\hline 43 & 0.049547 & 0.036069 & 0.021756 \\
\hline 44 & 0.044543 & 0.038417 & 0.031514 \\
\hline 45 & 0.040923 & 0.039887 & 0.037127 \\
\hline 46 & -0.083609 & -0.089603 & -0.098855 \\
\hline 47 & 0.046255 & 0.041632 & 0.037155 \\
\hline 48 & 0.057447 & 0.038061 & 0.019393 \\
\hline 49 & -0.082821 & -0.089814 & -0.100046 \\
\hline 50 & 0.057486 & 0.038080 & 0.018217 \\
\hline 51 & 0.039831 & 0.042691 & 0.040468 \\
\hline 52 & 0.052121 & 0.040578 & 0.029256 \\
\hline 53 & 0.017406 & 0.000852 & -0.020148 \\
\hline 54 & -0.004518 & -0.029016 & -0.060802 \\
\hline 55 & 0.064263 & 0.049505 & 0.031908 \\
\hline 56 & -0.003251 & -0.009163 & -0.028217 \\
\hline 57 & 0.028279 & -0.012766 & -0.047212 \\
\hline 58 & -0.015471 & -0.039281 & -0.063385 \\
\hline 59 & 0.069401 & 0.049597 & 0.031467 \\
\hline 60 & 0.032272 & -0.046990 & -0.115948 \\
\hline
\end{tabular}




\begin{tabular}{rrrr}
\hline 61 & 0.079963 & 0.045302 & 0.010180 \\
62 & -0.012840 & -0.047294 & -0.084501 \\
63 & 0.074408 & 0.045632 & 0.016346 \\
64 & 0.019351 & -0.040857 & -0.086072 \\
65 & 0.075162 & 0.050323 & 0.024874 \\
66 & -0.012183 & -0.015863 & -0.023090 \\
67 & 0.122832 & 0.010704 & -0.103894 \\
68 & -0.010212 & -0.021843 & -0.025473 \\
69 & 0.033220 & -0.028300 & -0.090364 \\
70 & 0.069335 & 0.049098 & 0.026864 \\
71 & 0.012613 & -0.006389 & -0.017872 \\
72 & -0.024164 & -0.017272 & -0.006787 \\
73 & 0.014528 & 0.000052 & -0.007537 \\
74 & -0.036601 & -0.057218 & -0.075782 \\
75 & 0.061114 & 0.042262 & 0.025646 \\
76 & -0.004804 & -0.047889 & -0.082799 \\
77 & -0.034391 & -0.057306 & -0.079000 \\
78 & 0.063366 & 0.042184 & 0.022896 \\
79 & 0.017251 & -0.000267 & -0.010854 \\
80 & 0.041852 & 0.042174 & 0.037770 \\
81 & 0.070106 & 0.045627 & 0.022686 \\
82 & 0.422340 & 0.414828 & 0.403890 \\
83 & 0.030542 & 0.007240 & -0.022117 \\
84 & -0.056913 & -0.036753 & -0.016017 \\
85 & -0.122158 & -0.160376 & -0.203130 \\
86 & -0.189076 & -0.194486 & -0.200641 \\
87 & 0.039738 & 0.037171 & 0.033574 \\
88 & 0.040416 & 0.037420 & 0.032878 \\
89 & 0.050563 & 0.036526 & 0.021795 \\
90 & -0.188632 & -0.193625 & -0.199576 \\
91 & 0.037559 & 0.039184 & 0.038712 \\
92 & 0.043559 & 0.038254 & 0.031677 \\
93 & 0.049675 & 0.036062 & 0.021966 \\
94 & -0.187586 & -0.193405 & -0.199996 \\
95 & 0.049547 & 0.036069 & 0.021756 \\
96 & 0.044543 & 0.038417 & 0.031514 \\
97 & 0.040923 & 0.039887 & 0.037127 \\
98 & -0.083609 & -0.089603 & -0.098855 \\
99 & 0.046255 & 0.041632 & 0.037155 \\
100 & 0.057447 & 0.038061 & 0.019393 \\
101 & -0.082821 & -0.089814 & -0.100046 \\
102 & 0.057486 & 0.038080 & 0.018217 \\
103 & 0.039831 & 0.042691 & 0.040468 \\
104 & 0.052121 & 0.040578 & 0.029256 \\
\hline & & & \\
\hline 9
\end{tabular}




\section{References}

(1) Kozhemyakina, N. V.; Nuss, J.; Jansen, M. Z. Anorg. Allg. Chem. 2009, 635, 1355-1361.

(2) Rudebusch, G. E.; Zafra, J. L.; Jorner, K.; Fukuda, K.; Marshall, J. L.; Arrechea-Marcos, I.; Espejo, G. L.; Ortiz, R. P.; Gómez-García, C. J.; Zakharov, L. N.; Nakano, M.; Ottosson, H.; Casado, J.; Haley, M. M. Nat.Chem. 2016, 8, 753-759.

(3) SADABS, Bruker AXS. 2001.

(4) Sheldrick, G. M. SHELXTL, Version 6.14, University of Göttingen, Germany 2000.

(5) Spek, A. L. Acta Cryst. 2015, C71, 9-19.

(6) Baonza, V. G.; Taravillo, M.; Arencibia, A.; Cáceres, M.; Núñez, J. J. Raman Spectrosc. 2003, 34, 264-270.

(7) del Corro, E.; González, J.; Taravillo, M.; Flahaut, E.; Baonza, V. G. Nano Lett. 2008, 8, 2215.

(8) Bannon, E.; Williams, Q. Phys. Chem. Minerals 2016, 43, 181-208.

(9) Zhao, L.; Baer, B. J.; Chronister, E. L. J. Phys. Chem. A 1999, 103, 1728-1733.

(10) Huan, W.; Zhang, J.; Berlie, A.; Qin, Z. X.; Zhao, X. M.; Zhang, J. B.; Tang, L. Y.; Luin, J.; Zhang, C.; Zhong, G. J.; Lin, H. Q.; Chen, X. J. J. Chem. Phys. 2013, 139, 10432. 